OPEN ACCESS

Edited by:

Tina Kyndt,

Ghent University, Belgium

Reviewed by:

Sotiris Tjamos,

Agricultural University of Athens, Greece

Cris Argueso,

Colorado State University, United States

*Correspondence:

Steven J. Klosterman steve.klosterman@usda.gov Nikhilesh Dhar

ndhar@ucdavis.edu

Specialty section:

This article was submitted to Plant Pathogen Interactions,

a section of the journal

Frontiers in Plant Science

Received: 19 July 2020 Accepted: 12 October 2020 Published: 04 November 2020

Citation:

Dhar N, Chen J-Y, Subbarao KV and Klosterman SJ (2020)

Hormone Signaling and Its Interplay With Development and

Defense Responses in Verticillium-Plant Interactions.

Front. Plant Sci. 11:584997. doi: 10.3389/fp/s.2020.584997

\section{Hormone Signaling and Its Interplay With Development and Defense Responses in Verticillium-Plant Interactions}

\author{
Nikhilesh Dhar ${ }^{1 *}$, Jie-Yin Chen ${ }^{2}$, Krishna V. Subbarao ${ }^{1}$ and Steven J. Klosterman ${ }^{3 *}$ \\ ${ }^{1}$ Department of Plant Pathology, University of California, Davis, Salinas, CA, United States, ${ }^{2}$ Department of Plant Pathology, \\ Institute of Plant Protection, Chinese Academy of Agricultural Sciences, Beijing, China, ${ }^{3}$ United States Department of \\ Agriculture, Agricultural Research Service, Salinas, CA, United States
}

Soilborne plant pathogenic species in the fungal genus Verticillium cause destructive Verticillium wilt disease on economically important crops worldwide. Since $R$ gene-mediated resistance is only effective against race 1 of $V$. dahliae, fortification of plant basal resistance along with cultural practices are essential to combat Verticillium wilts. Plant hormones involved in cell signaling impact defense responses and development, an understanding of which may provide useful solutions incorporating aspects of basal defense. In this review, we examine the current knowledge of the interplay between plant hormones, salicylic acid, jasmonic acid, ethylene, brassinosteroids, cytokinin, gibberellic acid, auxin, and nitric oxide, and the defense responses and signaling pathways that contribute to resistance and susceptibility in Verticillium-host interactions. Though we make connections where possible to non-model systems, the emphasis is placed on Arabidopsis- $V$. dahliae and $V$. longisporum interactions since much of the research on this interplay is focused on these systems. An understanding of hormone signaling in Verticillium-host interactions will help to determine the molecular basis of Verticillium wilt progression in the host and potentially provide insight on alternative approaches for disease management.

Keywords: Verticillium wilt, Verticillium-host interaction, plant defense response, growth, development, phytohormones, hormone signaling pathways, cross-talk

\section{INTRODUCTION}

The genus Verticillium is composed of 10 species that cause Verticillium wilt on over 200 economically important plant species worldwide (Bhat and Subbarao, 1999; Klosterman et al., 2009; Inderbitzin et al., 2011; Inderbitzin and Subbarao, 2014). Verticillium wilt results in extensive yield or plant losses in many crops, including cotton, potato, tomato, eggplant, lettuce, spinach, alfalfa, strawberry, oilseed rape, canola, sunflower, olive, and also numerous dicotyledonous trees and shrubs of ornamental value (Fradin and Thomma, 2006; Klosterman et al., 2009). Verticillium dahliae, the most destructive of the 10 species within the genus Verticillium (Inderbitzin et al., 2011; Inderbitzin and Subbarao, 2014), is a soilborne pathogen that produces spores (conidia) during mycelial growth and growth within the xylem, and microsclerotia, the 
thick-walled, and melanized dark brown to black structures for long-term survival in the soil environment (Short et al., 2015). Seed borne transmission of the microsclerotia has also been demonstrated (Vallad et al., 2005) and the potential pathogen spread via infested seed is low in case of lettuce but can occur on a transcontinental scale with infested spinach seed (Atallah et al., 2010; Short et al., 2015). In response to host root exudates, microsclerotia germinate and infect the host roots by direct penetration (Fradin and Thomma, 2006; Klosterman et al., 2009). Once the pathogen reaches the xylem, it systemically spreads within the host (Figure 1).

Resistance to Verticillium wilt is mediated by a dominant gene in several hosts, including tomato, sunflower, potato, and lettuce, among others (Hayes et al., 2011), but the pathogen eventually overcomes this resistance. Based on the response of differential host cultivars, $V$. dahliae has been divided into two races (Vallad et al., 2006; Maruthachalam et al., 2010; Sandoya et al., 2016) and an additional race was described on tomato (Usami et al., 2017). Races 1 and 2 were subsequently correlated with nondefoliating or defoliating phenotypes on cotton $\mathrm{Hu}$ et al., 2015), but this correlation is not perfect as many nondefoliating strains belong to a race different from race 1 (Zhang et al., 2019). Identified resistance genes have been incorporated into tomato resulting in control of race 1 (Diwan et al., 1999; Kawchuk et al., 2001; Fradin et al., 2009). Race 1 resistance in lettuce has been identified and efforts are currently underway to incorporate this resistance into elite cultivars (Hayes et al., 2007, 2011; Sandoya et al., 2016). However, this racedependent host resistance in tomato was compromised within a few years following its deployment (Grogan et al., 1979). Absence of safe and cost-effective control methods warrant the development of novel disease management techniques. Effectors of $V$. dahliae and their subsequent host defense components in plants have been identified (Fradin et al., 2011; de Jonge et al., 2012) and are important in the fight against Verticillium wilts. Thus, several approaches, including a thorough understanding of the signaling networks and discovery of novel genes that confer quantitative disease resistance against this pathogen are currently being explored.

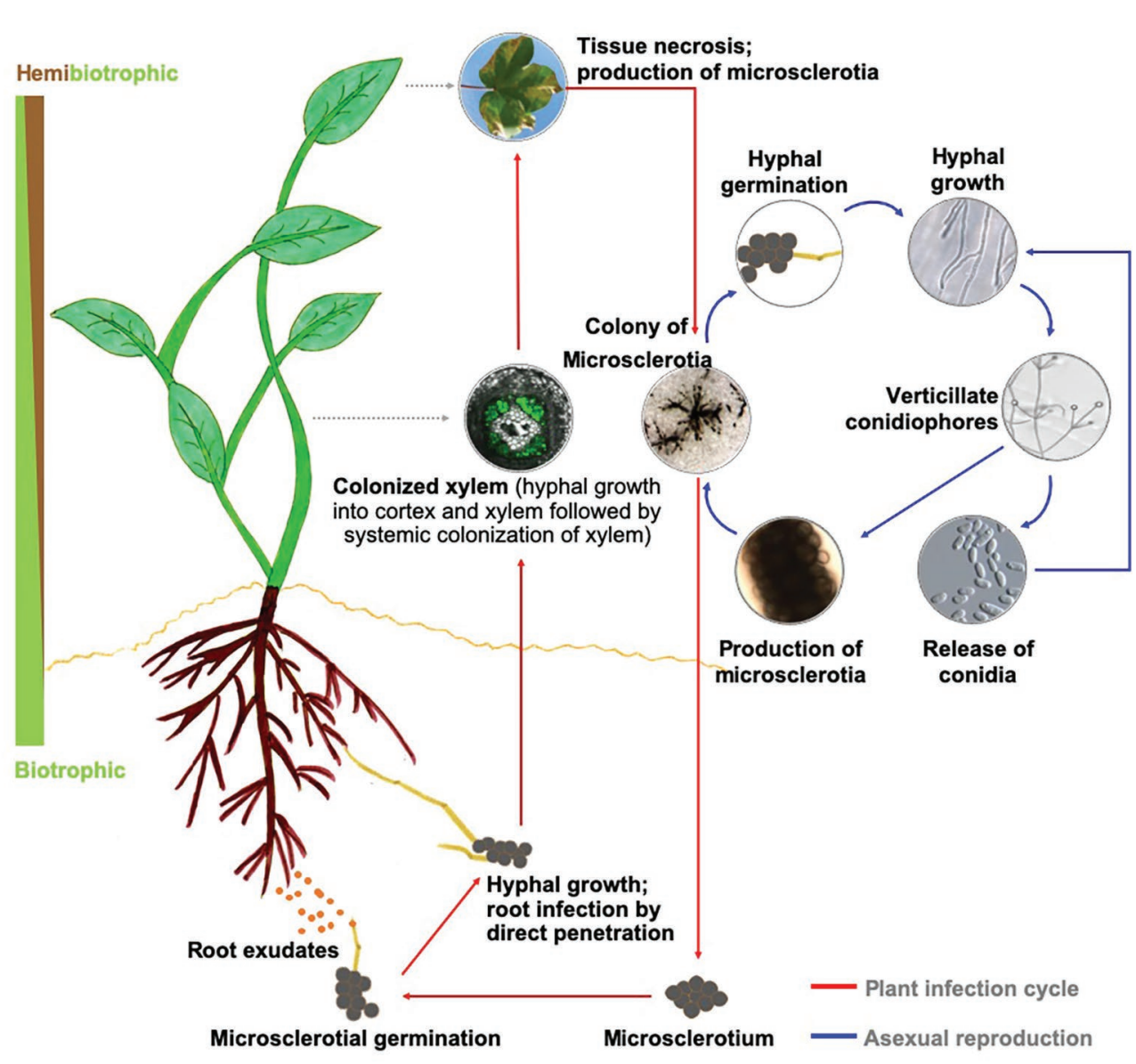

FIGURE 1 | Disease cycle in a generic host and biology of Verticillium dahliae. Upon perceiving signals in host root exudates, microsclerotia of Verticillium sp. germinate, and the resulting hyphae enter the plant through direct penetration or natural openings and progresses to colonize the xylem. During this biotrophic phase of its life cycle, the pathogen utilizes the host nutrients to propagate and achieves systemic spread in the host xylem. As the rapidly expanding pathogen overtakes the host defenses, the infected plant succumbs to the disease and eventually dies. This period in the infection cycle also overlaps with establishing the necrotrophic phase of Verticillium sp., during which the pathogen actively reproduces. As the host nutrient availability status recedes, the fungus forms microsclerotia for long-term survival of the pathogen. 
Plant hormones are a class of chemical compounds similar to animal hormones that are required in minute quantities to regulate growth and development. Plant hormones also play a central role in regulating defense responses against abiotic and biotic stresses, thereby integrating various aspects of plant health. Due to the sessile nature of plants and the central role hormones play in connecting the signaling networks governing an array of processes, precise execution of signal output is required for the healthy plant growth, successful completion of its life cycle culminating with the production of seeds for subsequent generations. External stimuli such as abiotic and biotic factors, including environmental factors, microbes, pests, and herbivores, affect not only the hormone signaling networks but also the cellular hormone levels. In addition to the classical plant hormones such as ethylene (ET), auxin (AUX), cytokinin (CTK), gibberellic acid (GA), and abscisic acid (ABA), research in the past two decades has led to the discovery of new hormones such as salicylic acid (SA), jasmonic acid (JA), brassinosteroids (BR), nitric oxide (NO), strigolactones (STR), karrikins (KAR), and others including peptide hormones and polyamines (Shigenaga and Argueso, 2016; Berens et al., 2017). Interestingly, many of these hormones are produced by plant pathogenic microbes as secondary metabolites and some pathogens may overcome host defense responses with a combination of effectors and interference with host hormone signaling. In contrast, some effectors produced by plant pathogens directly influence hormone levels or the hormone signaling network components to manipulate host resources to their advantage (Shigenaga and Argueso, 2016; Berens et al., 2017). The nature of these interactions determines the outcome of host-pathogen interactions while also affecting the hormone signaling pathways that integrate processes such as nutrient acquisition, defense, growth, and development.

Plant-microbe interactions can be manipulated by the application of plant hormones directly or by interference in hormone signaling processes to assess responses that affect plant productivity as well as defense responses (Delaney et al., 1994; Li et al., 1996; Tjamos et al., 2005; Johansson et al., 2006). A better understanding of plant hormonal responses to Verticillium spp. in both susceptible and resistant interactions will aid in effective management of Verticillium wilt while maintaining crop yields. In this review, we examine historical and novel developments on plant hormonal changes in response to pathogenic Verticillium species. We present the known host genetic components, defense strategies, and molecular mechanisms underlying defense responses in relation to hormone signaling pathways and identify research areas for the future.

\section{HORMONE SIGNALING DURING VERTICILLIUM INFECTION}

\section{Salicylic Acid Signaling}

SA plays a central role in plant defense responses including systemic acquired resistance (SAR; Delaney et al., 1994; Cao et al., 1997; Vlot et al., 2009). Though SA-mediated pathways regulate defense against biotrophic and hemibiotrophic pathogens, they play ancillary roles against necrotrophic pathogens (Glazebrook, 2005). SA pathway components are required for resistance against Verticillium spp. in Arabidopsis, tomato, and cotton (Johansson et al., 2006; Fradin et al., 2009; Gao et al., 2011; Dhar et al., 2019). In Arabidopsis-V. longisporum interactions, SA levels have been shown to be augmented in an Arabidopsis genotype-specific manner following inoculations with the pathogen (Haffner et al., 2014). Similarly, levels of SA and its glucoside (SAG) were elevated in the root xylem sap, hypocotyl, and shoots in Brassica napus following V. longisporum inoculations, while JA and ABA levels in the xylem sap remained unaffected (Ratzinger et al., 2009). While confirming these results, Zheng et al. (2019) further demonstrated that the introduction of bacterial NahG (encoding salicylate hydroxylase that converts SA to catechol) into the host plant depleted SA and increased susceptibility to $V$. longisporum. Thus, a threshold SA level may be required to confer resistance against this pathogen.

The phenylpropanoid pathway is the major contributor to SA biosynthesis in potato. The expression of phenylalanine ammonialyase 1 (PAL1) and phenylalanine ammonia-lyase 2 (PAL2) is upregulated in potato following $V$. dahliae infection. The response of these genes, along with other SA signaling genes such as pathogenesis related genes including $P R 1, P R 2$, and $P R 5$ are induced strongly in the moderately resistant cultivars compared to the susceptible cultivars (Derksen et al., 2013). Furthermore, on cotton calli, exogenous SA application led to the protection of callus cells against the deleterious effects of $V$. dahliae (VD) toxin (Zhen and Li, 2004). A genome-wide association study (GWAS) in cotton determined that the cotton glutathione S-transferase 9 (GaGSTF9) was a positive regulator of defense against Verticillium wilt, following enhanced SA signaling in response to the pathogen (Gong et al., 2017). Not only are GSTs inducible by exogenous SA application, but also the OxGSTF9 plants showed higher induction of SA levels in planta upon challenge with $V$. dahliae, indicating that GSTs play a significant role in SA-mediated defense in $V$. dahliae-cotton interactions.

The plant nonrace specific disease resistance 1 (NDR1) gene is central to SA-mediated defense responses and is required for resistance against plant pathogenic bacteria, fungi, and nematodes (Century et al., 1995; Gao et al., 2011; Dhar et al., 2019). NDR1 is required for a reactive oxygen species (ROS)-dependent hypersensitive response (HR) and the accumulation of SA in response to pathogens (Century et al., 1995). Both NDR1 and enhanced disease susceptibility 1 (EDS1) signaling pathways are required for the signaling cascade downstream of the Verticillium race1 resistance (Ve1)-mediated defense responses in tomato, even though these SA signaling components mediate defense responses to two different classes of nucleotide-binding leucine-rich repeat (NBS-LRR) type defense proteins in plants (Aarts et al., 1998; Hu et al., 2005; Fradin et al., 2009). GbEDS1, the ortholog of A. thaliana EDS1 in cotton, is induced by both Verticillium infection and SA. While ectopic expression of GbEDS1 led to increased resistance with increased levels of SA and the ROS hydrogen peroxide, silencing of the gene produced the opposite effect (Yan et al., 2016). Following $V$. dahliae infection, the levels of AtEDS1, AtNDR1, and BRI1-associated receptor kinase 1 (AtBAK1) were significantly 
upregulated in transgenic Arabidopsis plants ectopically expressing the cotton GbEDS1, while GbEDS1-silenced cotton plants showed the opposite trend. Although both EDS1 and NDR1 are known to regulate different classes of $R$ gene-mediated resistance pathways, both of these genetic components are required for resistance against $V$. dahliae in an SA-associated manner (Aarts et al., 1998; Fradin et al., 2009; Yan et al., 2016).

Furthermore, a mutation in the allotetraploid cotton 5-aminolevulinic acid dehydratase (ALAD) gene, encoding an enzyme that localizes into the chloroplast and functions at an early step in chlorophyll and heme biosynthesis, resulted in high levels of ROS, SA, and constitutive expression of PRs leading to increased resistance against $V$. dahliae. High levels of ROS via auto oxidative decomposition in a synergistic amplification loop, aided by upregulation of positive regulators of SA biosynthetic genes, including EDS1, phytoalexin deficient 4 (PAD4), and PAL were proposed to underlie the increased tolerance to $V$. dahliae (Chai et al., 2017).

Further evidence for SA-mediated defense response against Verticillium infection was described by Liu et al. (2014), who showed that Arabidopsis plants expressing the Pseudomonas putida NahG resulted in increased susceptibility of the plants. The authors inferred that suppressing plant SA-mediated defense responses downstream of SA biosynthesis may be the cause of this increased susceptibility. In the V. longisporum-infected oilseed-rape Brassica napus system as well, the SA-mediated defense responses were required for resistance (Zheng et al., 2019). While increased levels of SA were observed in the resistant cultivar compared with the susceptible cultivar in early asymptomatic stages, this difference was nullified at later stages of infection. As with cotton-Verticillium interactions, $V$. longisporum-treated transgenic B. napus plants expressing the SA catabolic NahG resulted in increased susceptibility to the pathogen (Zheng et al., 2019), reiterating the importance of SA-mediated responses in host resistance against Verticillium.

In yet another study involving Arabidopsis, mutations in genes for SA biosynthesis and accumulation did not result in a significant loss of resistance to $V$. dahliae; however, a mutation in the SA receptor nonexpresser of $P R$ genes 1 (NPR1) resulted in the destabilization of this protein leading to susceptibility (Johansson et al., 2006), indicating that SA perception is equally important. Though Arabidopsis plants with a mutation in NPR1 increased susceptibility to Verticillium (Johansson et al., 2006), cotton plants with ectopic expression of AtNPR1 heightened resistance to only the non-defoliating strains of $V$. dahliae; with resistance to the defoliating strain remaining unaltered (Parkhi et al., 2010). Similarly, ectopic expression of NPR1 from wild eggplant Solanum torvum (StoNPR1) in a susceptible potato cultivar resulted in increased resistance to Verticillium, whereas transgenic StoNPR1RNAi expressing potato plants showed increased susceptibility (Deng-Wei et al., 2014). Thus, fortification of resistance to Verticillium is likely dependent on the levels of NPR1 as inferred from studies on multiple host species. Root colonization by the plant-growth-promoting rhizobacterium (PGPR) Paenibacillus alvei strain K165 activates induced systemic resistance (ISR) in a SA-dependent manner to increase resistance in Arabidopsis. The ISR activity of K165 was dependent on SA pathway biosynthesis genes salicylic acid induction deficient 1 (SID1), salicylic acid induction deficient 2 (SID2), and the receptor gene NPR1, since a loss-of-function of these genes abrogated the effect in K165treated mutant plants (Tjamos et al., 2005).

While multiple genetic components, biochemical pathways, metabolites, and the SA signaling components play important roles in modulating defense responses against Verticillium spp., transcriptional reprograming after infection is also associated with SA signaling. Changes in plant transcription and translation machinery by pathogen infection are routinely correlated with alterations of phytohormone levels. A 60 S ribosomal subunit protein (RPL18) necessary for intracellular protein biosynthesis was shown to regulate resistance against Verticillium in both cotton and Arabidopsis in a SA-dependent manner (Gong et al., 2017). While the cotton GaRPL18 was strongly and rapidly induced by the application of SA, levels of SA were significantly lower in the plants where GaRPL18 was silenced. Additionally, a strong induction of the SA-related genes but not the JA or ET pathway genes was found in $V$. dahliae-challenged Arabidopsis plants over-expressing GaRPL18. In contrast, pretreatment of such plants with SA resulted in increased resistance to the pathogen in these transgenic plants relative to the treated wild type plants (Gong et al., 2017).

Many pathogens employ effector molecules that alter the hormone levels in plants as an infection strategy (Shigenaga and Argueso, 2016). Consequently, a search for effector proteins that might be employed by Verticillium spp. to suppress SA levels in the host led to the discovery of a V. dahliae isochorismate synthase 1 (VdIsc1) that was upregulated during early infection stages (Liu et al., 2014). When cotton plants were inoculated with a VdIsc1 deletion mutant, the levels of the host SA and SAG levels, as well as that of the SA marker, $P R 1$, were significantly upregulated. Another secretory protein $V$. dahliae secretory protein 41 (VdSCP41) targets the master immune regulators cam-binding protein 60-like $G(C B P 60 g)$ and $S A R$ deficiency 1 (SARD1) that regulate the SA-mediated defense response sector in multiple plant species, including Arabidopsis and cotton (Qin et al., 2018). While CBP60g and SARD1 bind directly to the promoters of SID2, enhanced disease susceptibility 5 (EDS5), and NPR1, the intracellular fungal effector VdSCP41 binds to these upstream transcription factors (TFs; CBP60g and $S A R D 1$ ) and regulates their activity inside the host cell (Qin et al., 2018). Thus, SA signaling plays a significant role during plant-Verticillium interactions. SA perception through NPR1 and pathogen-mediated alterations in host SA levels seem to determine the outcome of resistance against this pathogen.

\section{Role of Jasmonic Acid Signaling}

Jasmonic acid and the related jasmonates are a group of plant hormones that play a role in plant response to biotic stress, abiotic stresses, and plant developmental processes and share structural similarity to the prostaglandin class of metazoan hormones in animals (Chini et al., 2007). JA, which is a major constituent of the flower scent of jasmine, is synthesized in chloroplasts from membrane lipids in plants through the octadecanoid pathway and is perceived by coronatine-insensitive 1 (COI1; Berens et al., 2017). JA plays a major role in defense 
against the necrotrophic pathogens, herbivores, insect pests and wounding, as well as in plant reproduction, including pollen fertility (Xie et al., 1998; Berens et al., 2017).

Pretreatment of cotton plants with methyl-jasmonate (MeJA) before infection with $V$. dahliae confers resistance. This resistance was attributed to the activation of basal resistance mechanisms in the host (Li et al., 1996). Subsequent work established a role for JA and ET signaling pathway components in resistance against V. longisporum in Arabidopsis (Johansson et al., 2006), while brassinosteroid (BR) and JA regulate resistance against this pathogen in cotton (Gao et al., 2013a). JA biosynthetic genes, including lipoxygenase 3 (LOX3), lipoxygenase 4 (LOX4), and oxophytodienoate-reductase 3 (OPR3), were induced within $24 \mathrm{~h}$ post-treatment with $V$. dahliae. However, differences in the levels of active JA and jasmonoyl isoleucine (JA-Ile) were detected only 3 weeks post-inoculation, indicating that the upregulation of the genes involved in pathogen mediated phytohormone levels are induced early in the infection when the fungus was in the pre-vascular growth phase inside the host root tissue (Scholz et al., 2018). JA pathway mutants like jasmonate resistant 1 (jar1), coronatine insensitive 1 (coil), and cytochrome P450_family 94_subfamily B_polypeptide 3 (cyp94B3) exhibited reduced colonization and lower fungal biomass in roots and thus were resistant to V. dahliae (Scholz et al., 2018). Based on these results, the authors hypothesized that the pathogen might require JA components to promote necrosis type cell death in the later phases of infection, while an absence of JA might lead to activation of SA-mediated responses required for host resistance by prolonging the biotrophic phase of $V$. dahliae.

A nuclear localizing TIR-NBS-LRR protein from a cotton resistant cultivar with homology to Arabidopsis dominant suppressor of camta3 1 (DSC1) and tobacco $N g N$ regulates resistance to $V$. dahliae in both Arabidopsis and cotton. While GhDSC1 was specifically upregulated by $V$. dahliae in the resistant cultivar, it was strongly induced by the application of JA but not to the same extent by any other hormone tested. While the Arabidopsis dsc1 mutant was susceptible to V. dahliae, ectopic expression of GhDSC1 led to increased resistance, and GhDSC1 complemented the susceptible phenotype of the Arabidopsis dsc1 mutant. DSC1 mediated resistance to V. dahliae involved the accumulation of ROS. Studies using transgenic GhDSC1 ectopic expression showed that it complemented the host defense response to the pathogen, which was activated through the JA signaling pathway. Furthermore, the activation of DSC1 was negatively regulated by calmodulin-binding transcription activator 3 (GhCAMTA3), which shares similar activation kinetics and regulatory stimuli with those of DSC1, leading the authors to hypothesize that the function of these two defense regulators may be coupled through modulation of JA signaling pathway in cotton (Li et al., 2019).

Many components of plant metabolic pathways contribute to defense through regulating the hormone-mediated responses. Lignin biosynthesis, a branch of the phenylpropanoid metabolism, reinforces plant cell walls, which serve as a natural barrier against pathogen penetration, and the secondary metabolic products associated with lignin production are upregulated in Verticillium-tomato interactions (Hu et al., 2018a, 2019). The cotton LACCASE 1 (GhLac1) functions as a lignin polymerization enzyme and was strongly induced by $V$. dahliae and JA, but not SA, in the roots of the cotton plants (Hu et al., 2018a, 2019). Loss-of function in this genetic component in cotton led to a redirection of the metabolic flux resulting in the accumulation of JA and JA-Ile that was further enhanced during infection with $V$. dahliae. This increased resistance against $V$. dahliae and cotton bollworm (Hu et al., 2018a). Another genetic component that affects the lignin content and JA signaling is the cotton umecyanin-like 1 (GhUMC1) encoding a blue copper-binding protein. While this gene is highly expressed in the root tissues, infection by $V$. dahliae leads to the downregulation of the gene. Moreover, the JA application upregulated this gene, but the silencing of this gene led to reduced expression levels of genes involved in JA biosynthesis and signaling in general (LOXs, MCY2, and OPR3). The GhUMC1 silenced plants were compromised in their ability to induce oxidative burst and synthesize lignin in response to $V$. dahliae, resulting in enhanced susceptibility (Zhu et al., 2018).

Some plant genes that have more indirect roles in SA and JA regulation act as regulators of defense responses against Verticillium infection. The peroxisome-localized cotton calciumdependent protein kinase 33 (GhCPK33) was specifically induced by $V$. dahliae strain V991 and served as a negative regulator of resistance. Induction of this gene resulted in the destabilization of the JA biosynthesis gene GhOPR3, and impaired the JA dependent defenses and rendered the host susceptible (Hu et al., 2018b). Another cotton cytochrome P450 CYP82D family gene, silenceinduced stem necrosis (GhSSN), was induced by JA and V. dahliae in the roots of susceptible cotton but was downregulated in the resistant line. Furthermore, silencing of this gene induced HR-like lesion mimic mutants that induced hyperaccumulation of JA, which in turn led to the suppression of the SA signaling and enhanced resistance against $V$. dahliae, suggesting that JA rather than SA plays a critical role in resistance against $V$. dahliae (Sun et al., 2014). The cotton stearoyl acyl-carrier-protein desaturase suppressor of SA insensitive 2 (GbSSI2) was strongly induced in the resistant $G$. barbadense cv7124 following $V$. dahliae infection. While the silencing of the GbSSI2 led to increased susceptibility to Verticillium accompanied by downregulation of the JA biosynthesis and signaling components, there was a concomitant increase in the endogenous SA levels (Gao et al., 2013a). The above results indicate that $V$. dahliae infection activates the JA signaling pathway in cotton, and a loss of GbSSI2 function led to the suppression of defense responses and the loss of resistance (Gao et al., 2013b).

The mediator complex is a conserved multiprotein cofactor of RNA polymerase II and regulates transcription. It consists of 20-30 subunits that form four mediator subcomplexes, which in turn is subjected to dynamic regulation intertwined with hormone signaling. Mediator subunits influence multiple plant processes like development, flowering, non-coding RNA processing, secondary metabolism, and defense response to various abiotic and biotic stresses (An and Mou, 2013; Li et al., 2018b). The expression of one such cotton cyclin-dependent kinase $E$ (GhCDKE), a subunit of the cotton mediator complex, is induced by $V$. dahliae infection and JA treatment. Not only were JA-mediated responses compromised in the CDKE-silenced cotton lines, but also the GhCDKE over-expressing Arabidopsis plants were more sensitive to JA, indicating that GhCDKE functions 
in the JA signaling pathway. The silencing of GhCDKE in cotton led to enhanced susceptibility to $V$. dahliae, while its ectopic expression in Arabidopsis led to enhanced resistance against the pathogen in a JA-dependent manner (Li et al., 2018b).

Transcriptome profiling of cotton plants infected with V. dahliae identified homeodomain transcription factor 1 (HDTF1), a negative regulator of JA signaling that is preferentially expressed in the leaf tissues. This gene is downregulated by $V$. dahliae infection and MeJA treatment while it is induced by SA treatment. The silencing of HDTF1 increased the expression of genes involved in JA biosynthesis and accumulation, leading to activation of JA-mediated signaling, though it was not accompanied by an alteration in SA accumulation or SA signaling. Thus, increased resistance of cotton to $V$. dahliae and $B$. cinerea in the HDTF1-silenced plants was attributed to enhanced JA signaling (Gao et al., 2016).

Cotton homeobox 12 (GhHB12) encodes a plant-specific homeodomain-leucine zipper (HD-ZIP) family protein that affects various plant developmental processes and is induced by $V$. dahliae infection and MeJA application. GhHB12 over-expressing plants displayed increased susceptibility to $V$. dahliae, which correlated with the stronger suppression of the JA-responsive, defense-related genes jasmonate-zim-domain protein 2 (GhJAZ2) and pathogenesis-related 3 (GhPR3), as well as decreased lignin content in the stem of infected cotton plants (He et al., 2018a).

Another transcription factor that represses JA-mediated defense response in response to $V$. dahliae infection of cotton is the plant-specific NAC transcription family member ATAF1. Though GhATAF1 expression increased upon treatment with SA and JA in conjunction with $V$. dahliae, over-expression of ATAF1 in cotton increased wilting and foliar discoloration indicative of increased susceptibility to the pathogen. Suppression of the JA signal components (including GhLOX1, GhMYC2, GhPR3, and GhPR4) by $V$. dahliae infection was accompanied by a concomitant increase in the SA pathway genes (including GhNDR1, GhNPR1, GhPR1, and GhPR5), highlighting a branch of defense signaling that involves reciprocal regulation of JA-SA mediated defense responses during Verticillium infection (He et al., 2016). The application of JA not only enhanced resistance against Verticillium but also inversely affected the growth of cotton plants (Li et al., 2014). Thus, hormonal signaling during pathogen infection could lead to transcriptional reprograming during defense responses to Verticillium in cotton and Arabidopsis.

Though both SA and JA signaling play a role in defense against Verticillium sp., the SA-mediated response appears to take a predominant role during the initial biotrophic phase of infection. During the necrotrophic phase, however, JA-mediated defenses play an active role, indicating that the host defense mechanism has evolved as a layered temporospatial response to counter or restrict the damages from the systemic pathogen spread within the host, followed by a strategic switch in its lifestyle during the subsequent progression of Verticillium wilt.

\section{Role of Ethylene Signaling}

The gaseous plant hormone ET plays a central role in senescence and defense responses to necrotrophic pathogens (Broekaert et al., 2006). Hence, the Verticillium wilt-associated chlorosis/yellowing of the foliage from the loss of chlorophyll prompted investigations into the role of ET signaling during Verticillium infection. Most studies on the role of ET in Verticillium wilt involve tomato, cotton, and Arabidopsis. Elevated ET levels were observed in tomato plants coinciding with the onset of symptoms (Cronshaw and Pegg, 1976). The production of ET was especially enhanced in the susceptible tomato lines by the culture filtrates fraction that were enriched in protein and pectolytic enzymes (Pegg and Cronshaw, 1976). While ET production was stimulated by only the protein/enzyme fraction of culture eluates, increased wilting and necrosis was observed with other eluted fractions, i.e., containing pectolytic enzyme activity or inactive polysaccharide in plants pretreated with ET. Taken together, these results led to the proposition that ET may act as a toxin synergist in Verticillium wilt of tomato (Pegg, 1976; Pegg and Cronshaw, 1976).

Studies in potato confirmed the role of ET in Verticillium pathology. Exposure of potato plants to a low-molecular-mass Verticillium toxin from the culture filtrate of $V$. albo-atrum $(=V$. nonalfalfae $)$ revealed correlation of susceptibility to $V d$ toxin with the differential production of ET. Susceptible cultivars produced more ET than tolerant potato cultivars. Inhibiting host ET signaling (perception/biosynthesis) by pharmacological treatments abrogated the toxin-induced and ET-mediated symptom development in the host, proving the involvement of ET in Verticillium wilt (Mansoori and Smith, 2005). Additionally, a necrosis- and ET-inducing protein $V$. dahliae necrosis-and ethylene-inducing protein (VdNEP) protein from a cotton isolate of $V$. dahliae activated both SA- and ET-JA-dependent defense pathways in Arabidopsis while strongly inducing the expression of the host 1-aminocyclopropane-1-carboxylic acid synthase 6 (ACS6) gene encoding the ET biosynthetic enzyme 1-aminocyclopropane-1-carboxylate synthase (Wang et al., 2004).

Genome-wide analysis of the pathogen later revealed the existence of the necrosis- and ET-inducing-like protein (NLP) family of conserved fungal effectors, of which eight were predicted in V. dahliae (Klosterman et al., 2011; Santhanam et al., 2013). Two members of this family (NLP1 and NLP2) expressed inside the host following infection exhibit cytotoxic activity. Furthermore, loss of function of these genes led to defects in conidiophore and aerial hyphae in the pathogen (Santhanam et al., 2013). Additionally, two members of a VdNLP family (VdNLP1 and VdNLP2) of cytolytic toxins induce ROS and promote necrosis in cotton with the induction of SA/JA defense pathways as well as upregulation of ACS6 during $V$. dahliae infection of cotton (Zhou et al., 2012).

Components of the ET signaling pathway-ethylene insensitive 2 (EIN2), ethylene insensitive 4 (EIN4), and ethylene insensitive 6 (EIN6) in Arabidopsis positively regulate defense responses against V. longisporum (Johansson et al., 2006). This study also shed light on the fact that ET production followed pathogen infection and was independent of ET sensitivity of the host root system, though a higher resistance to the pathogen was observed in the ethylene resistant 1-1 (etr1-1) mutant. Although ET pretreatment did not compromise fungal growth on media, treatment of the pathogen-challenged host with the ethylene biosynthetic precursor 1-aminocyclopropane-1-carboxylic acid 
(ACC) resulted in a substantial increase in the fresh weight of the inoculated plants. Evidence for the involvement of ET signaling components in Verticillium wilt came from studies involving Never Ripe (SlNr) and ethylene resistant 4 (SIETR4) in tomato, wherein silencing of these ET receptor genes reduced disease incidence, severity, and reduced fungal biomass indicative of increased resistance to $V$. dahliae (Pantelides et al., 2010a). Impaired perception of ET in the Arabidopsis etr1-1 mutant led to a significant reduction in pathogen growth and increased resistance to $V$. dahliae (Pantelides et al., 2010b).

In cotton, an ethylene response factor-like gene (GbERF1like) is induced by $V$. dahliae along with the hormones MeJA and ET, respectively. The GbERF1-like protein acts as a positive regulator of defense against the pathogen by fortifying lignin synthesis upon pathogen challenge (Guo et al., 2016). A cotton major latex protein 28 (GhMPL28) interacted with the cotton ethylene response factor 6 (GhERF6) transcription factor facilitating its binding to the GCC-box element in the promoters of the defense-related genes and positively regulating defense against $V$. dahliae (Yang et al., 2015).

The role of ET during Verticillium infection has been contradictory, with some studies indicating a dual role for ET in resistance as well as the promotion of wilt. Studies involving different stages of infection and the timing of ET biosynthesis/ perception have elucidated the exact role of this hormone during Verticillium wilt symptom in tomato. Though symptom development and ET production rapidly increased in the first few days after $V$. dahliae inoculation of tomato, these declined thereafter (Robison et al., 2001a). A reduction in the pathogeninduced ET production in tomato plants treated with $V$. dahliae was achieved upon the application of the ET inhibitor aminoethoxy vinyl glycine (AVG) either before or at the time of inoculation. This led to greater resistance, reduced symptom severity, reduced disease, and greater fresh weight of the infected host (Robison et al., 2001a). Complimentary studies have shown that plants transformed with the bacterial ACC deaminase gene under root-specific or infection-induced promoters that inhibit ET synthesis, significantly reduced or delayed symptoms when challenged with $V$. dahliae. Tissue or defense-specific induction of this catabolic enzyme degraded the ET precursor ACC, resulting in reduced chlorosis and wilting, increased fresh weight, and overall larger plants (Robison et al., 2001b).

Another study examining the role of ACS genes found that the 1-aminocyclopropane-1-carboxylic acid synthase 2 (ACS2) and 1-aminocyclopropane-1-carboxylic acid synthase 6 (ACS6) genes were upregulated by 2 weeks post- $V$. dahliae infection in $A$. thaliana. The authors further demonstrated a lower endophytic level of $V$. dahliae in mutant plants since mutant plants were more resistant than the wild type plants in an ethylene dependent manner (Poulaki et al., 2020). While the production of ET by the host plant during early stages of $V$. dahliae infection results in the induction of defense responses, ET production post-infection seems to favor enhanced infection. More significantly, the results from this study demonstrate that components of ET biosynthesis, as well as the ET precursor ACC, play an important role during the progression of Verticillium wilts.
Though ET plays a significant role in the plant responses to Verticillium spp., the effect of ET signaling that determines the outcome of this interaction seems to depend on the timing, amplitude, and spatial nature of the perception, and relay of the signal during fungal pathogenesis. Perception of the pathogen seems to activate ET signaling along with the induction of ET biosynthesis by multiple Verticillium proteins and toxins, which in turn activates the defense responses and induce cell death to limit the pathogen at the site of infection during the early stages of infection. But in later stages of infection, during colonization and the switch to the necrotrophic phase, these responses might, in fact, aid in the establishment of the pathogen.

\section{Role of Brassinosteroid Signaling}

Brassinosteroids are group steroid hormones that are found in both plants and animals. In plants, BR is primarily involved in plant growth, cell differentiation, and developmental processes, including reproduction and senescence, but are most notable for their involvement in the photomorphogenesis of plants. While the receptors for plants are present on the cell surface, the receptors for the animal counterparts are transcription factors that belong to the nuclear receptor family (Wang et al., 2012). However, their role in defense responses to various pathogens has attracted further attention.

Long-term exposure to epibrassinolide reduced or eliminated symptoms of Verticillium infected tomato plants while shortterm exposure had no effect (Krishna, 2003; Roos et al., 2014). Analyses of cotton calli exposed to Verticillium suggests the involvement of BR in ameliorating Verticillium toxin-induced stress (Bibi et al., 2017) while BR signaling component BRI1associated receptor kinase (BAK1) was shown to be a positive regulator of race-specific disease resistance against the pathogen in tomato (Fradin et al., 2009; Roos et al., 2014). Pretreatment of cotton calli rather than co-/post-treatment with epibrassinolide significantly negated the effects of $V d$ toxin in a pathogen-free model system with a significant increase in photosynthetic pigments and accompanying secondary metabolism (Bibi et al., 2017). A decrease in water and nutrient uptake caused by the clogging of the xylem by Verticillium cell wall degrading enzymes is known to induce osmotic stress during Verticillium pathogenesis (Bibi et al., 2014). A decline in photosynthesis caused by reduction in leaf surface area as well as the wilting of leaves causes a decline in the overall photosynthetic ability of the plant during Verticillium infection contributing to the loss of carbohydrate metabolites that could mitigate the effect of osmotic stress caused by the pathogen in the vascular tissues (Bowden et al., 1990; Hampton et al., 1990; Sadras et al., 2000). Plants try to counter this with an increase in the enzymes involved in carbohydrate metabolism, including sucrose phosphate synthase (SPS), vacuolar/cell wall-bound acid invertase (AI), and cytosolic sucrose synthase (SuSy; Bibi et al., 2014). While Vd toxin resulted in a slight increase in these enzymes, the application of epibrassinolide significantly elevated the level of these enzymes. Such induction was achieved by a relatively lower concentration of the hormone applied to the roots, while a higher concentration was required for foliar induction (Bibi et al., 2014). The effect of such osmotic stress is mitigated by the host in part by an 
increase in production of proline, glycine betaine, and soluble sugar (Goicoechea et al., 2000; Bibi et al., 2014). Further, the application of BR mitigated the growth inhibitory effect of a $V d$ toxin by reducing the damage to chlorophyll and enhancing the rate of photosynthesis as well as transpiration, resulting in increased root and shoot biomass (Bibi et al., 2014).

Disease resistance in cotton may in part depend on brassinolide signaling. $B A K 1$, which encodes an LRR-RLK that is associated with the BR receptor brassinosteroid insensitive 1 (BRI1) and belongs to a sub-family of receptor-like kinases (RLKs) supports this hypothesis. Loss-of-function of this gene not only led to cell death accompanied by the production of ROS but also resulted in resistance against the Verticillium wilt in Arabidopsis, tomato, and cotton showing that this signaling pathway is conserved across species (Fradin et al., 2009; Gao et al., 2013a; Roos et al., 2014). Yet another study utilizing proteomics analysis led to the discovery of crosstalk between the JA-BR pathways (Gao et al., 2013b). While this work highlighted that BR signaling was activated in the cotton-treated with Verticillium, it also showed that the disease resistance in cotton was fortified upon the application of brassinolide while activating the JA signaling pathway, indicating a positive correlation between these two hormone signaling pathways (Gao et al., 2013b).

Among the RLKs that are associated with BR signaling in plants is the suppressor of BIR1 (SOBIR1), which, as the name suggests, was discovered in a genetic screen on BAK1-interacting receptor-like-kinase 1 (BIR1). SOBIR1 is required for resistance against various pathogens and interacts with many receptorlike proteins (RLPs) as well as with BAK1 to influence cell death and defense response independent of BIR1 (Gao et al., 2009; Liu et al., 2016). While the cotton homolog of SOBIR1 gene (GbSOBIR1) is induced by $V$. dahliae, a reduction in the levels of expression of this BR pathway gene compromises the host resistance, and ectopic expression results in increased resistance to $V$. dahliae (Zhou et al., 2019). Although the expression of GbSOBIR1 was enhanced by the SA application, treatment with JA caused its downregulation. A previous study had found a positive effect between BR-induced resistance and activation of JA response upon challenge with the pathogen. Protein-protein interaction studies led to the discovery of a basic helix-loop-helix (bHLH) family transcriptional activator bHLH171, and further experiments showed that this protein is phosphorylated by GbSOBIR1 most likely at a serine residue that is required for its transcriptional activity (He et al., 2018b). The homolog of Arabidopsis SOBIR1 in tomato (SISOBIR1) is required for resistance to $V$. dahliae in a Ve1-dependent manner and interacted with a broad range of RLPs. Further, the silencing of this gene resulted in reduced Ve1 levels leading to impaired HR during Ve1-Ave interaction in the plant (Liebrand et al., 2013). Since both Vel and BAK1 are conserved across plant species and form receptor complexes while contributing to resistance against $V$. dahliae, these two receptor-like proteins may partner with each other in some capacity during host defense responses to Verticillium wilt (Gao et al., 2013b).

Two Verticillium glycoside hydrolase 12 (GH12) proteins $V$. dahliae endoglucanase 1 (VdEG1) and V. dahliae endoglucanase 3 (VdEG3) act as pathogen-associated molecular patterns and induce immunity-related cell death in tobacco. In conjunction with another Verticillium effector carbohydrate-binding module family 1 (CBM1) protein, these GH12 Verticillium proteins together manipulate host defense responses involving the $\mathrm{BR}$ signaling components BAK1 and SOBIR1. Further, VdEG1 was perceived through the LRR-RLP/SOBIR1/BAK1 complex while VdEG3 was perceived through the LRR-RLKs/BAK1 complex independently of their enzymatic activity, leading to activation of defense response and host cell death. Both were in turn suppressed by the pathogen effector VdCBM1 in a dose-dependent manner to promote infection in the host (Gui et al., 2017). Another Verticillium cutinase family protein, $V$. dahliae CUTINASE 11 (VdCUT11) is notably upregulated and functions as a major virulence factor during $V$. dahliae infection in both cotton and tobacco, resulting in significant loss of plant biomass. However, its recognition leading to activation of host defense response leading to host cell death and damage-associated molecular pattern-triggered immunity, in turn, is suppressed by the VdCBM1 effector in a BAK1- and SOBIR1-dependent manner in tobacco plants. This sequence of events thus aids in the establishment of Verticillium wilt in the susceptible host (Gui et al., 2018).

A Rab GTPase-activating protein 22 (RabGap22) from Arabidopsis was induced by $V$. longisporum and is required for defense against the pathogen. Further inquiry on the RabGap22 co-expressed genes revealed the role of somatic embryogenesis receptor-like kinase 3 (SERK3)/BAK1 as a positive regulator of defense responses. Mutation in BAK1 not only increased the susceptibility but also attenuated the RabGap22 expression in V. longisporum-challenged Arabidopsis plants (Roos et al., 2014). The susceptibility of the rabgap22-1 mutant to this pathogen could be rescued with an application of 24-epibrassinolide, further indicating the importance of this phytohormone signaling pathway in host defense response during Verticillium infections.

\section{Role of Cytokinin Signaling}

Since reduced CK levels are associated with yellowing of leaves resulting from loss of chlorophyll, which is a hallmark of senescence and progression of Verticillium wilt, many studies have focused on the correlation of this hormone with senescence and wilt in susceptible hosts. Early studies on cotton plants (Gossypium hirsutum) treated with a pathogenic strain of Verticillium spp. showed a significant decrease in the levels of $\mathrm{CK}$ in the tracheal fluid and in the extract from the aerial tissues including stem and leaves, following leaf symptom development (Misaghi et al., 1972). Consistently lower levels of CK also occur in tomato plants following the onset of Verticillium wilt symptoms (Patrick et al., 1977). Based on these results, it was proposed that a decrease in water potential in the root leads to a reduction in CK levels, which in turn underlies the visible yellowing of the leaves due to chlorosis and loss of pigments during Verticillium infection (Patrick et al., 1977).

Verticillium-induced premature senescence is accompanied by a decrease in the host CK levels and this occurs because of upregulation of CK-degrading enzymes (Reusche et al., 2013). 
The expression maxima of these CK degrading enzymes cytokinin oxidase (CKX1, CKX2, and CKX3) correlated with the pathogeninduced decrease in the plant CK pool, trans-zeatin [tZ], in particular, which is the most prevalent $\mathrm{CK}$ in the model plant Arabidopsis. Reduced CK level was thus predicted to be beneficial for a necrotrophic pathogen or during the necrotrophic phase in the life cycle of a pathogen like $V$. longisporum, which might be a strategy for efficient colonization of the leaves by active induction of senescence (Reusche et al., 2013). External application of a synthetic CK as well as inhibition of the CK degrading CKX activity led to reduced symptoms and proliferation of the fungus on the host (Reusche et al., 2013). Thus, active CK levels seem to play an essential role in mediating defense responses during Verticillium infection.

\section{Role of Gibberellic Acid Signaling}

Hyper elongation of the rice stem in response to Fusarium fujikori is a striking example of the manipulation of GA signaling by a pathogen (Kurosawa, 1926). The pathogen-derived (plant growth-promoting) gibberellin has been attributed as the cause of bakanae disease on rice (Studt et al., 2013). As another example of the manipulation of GA levels by a pathogen, the interaction of the viral RVD outer capsid protein P2 with a rice gibberellin biosynthesis gene ent-kaurene oxidase (KAO) led to a reduction in the levels of this hormone during infection of the rice dwarf virus (Zhu et al., 2005).

Challenge by a pathogen results in a "growth versus defense conundrum" in plants (Chini et al., 2007; Huot et al., 2014), including in Verticillium-host interactions (Dhar et al., 2019). In Verticillium wilt of oilseed rape, the transition to reproductive stage is critical for the spread of the pathogen and appearance of disease symptoms, while in cotton, the severity of Verticillium wilt increases in the field is concomitant with the transition to flowering ( $\mathrm{Li}$ et al., 2014). The cross-talk between the aspartate-glutamate-leucine-leucine-alanine (DELLA) proteins that are negative regulators of plant growth and jasmonate zim domain (JAZ) proteins that are negative regulators of plant defense response appears to be involved in these interactions. While DELLA proteins negatively regulate the GA signaling pathway involved in plant growth, JAZ proteins negatively regulate the JA signaling pathway involved in plant defense response (Hou et al., 2010). WRKY genes belong to a family of transcription factors that are involved in multiple processes, including growth, defense, and various hormone signaling. One such member of the family in cotton (GbWRKY1), isolated from a bacteriophage full-length cDNA library, is induced by $V$. dahliae as well as by the phytohormones SA, JA, and ET (Shu-Ling et al., 2012). The cotton GbWRKY1 has also been shown to be a negative regulator of defense response against Verticillium and Botrytis, most likely through the attenuating JA signaling pathway. GbWRKY1 is expressed upon Verticillium infection as well as JA application but also binds to the JAZ1 promoter. The overexpression of GbWRKY1 in Arabidopsis also led to early flowering. Steady availability of optimal resources, combined with their sessile nature, is a limiting factor in the physiological processes of plants. Thus, plants switch between the investment in growth and development, on the one hand, and defense responses to various biotic and abiotic factors on the other, resulting in the "growth versus defense trade-off" (Chini et al., 2007; Huot et al., 2014). Taken together, the work by Li et al. (2014) is indicative of growth - defense axis that is influenced by GbWRKY1, with development favored over the defense in an antagonistic interaction between the JA/GA pathways.

The well-characterized Arabidopsis ndr1-1 defense mutant (Century et al., 1995; Aarts et al., 1998), which confers increased susceptibility to Verticillium infection, was shown to display early flowering (Figure 2A top panel) linked to enhanced expression of GA biosynthesis genes, increased bioactive GA in planta as well as a reduction in the levels of DELLA components (Dhar et al., 2019). Because NDR1 is central to SA-mediated defense responses, the establishment of SAR, and the HR response against pathogens (Century et al., 1995; Aarts et al., 1998), this new finding raises the exciting possibility that increased susceptibility in $n d r 1-1$ might not only be due to its inability to accumulate SA but also due to the fact that enhanced GA signaling in this mutant might also contribute toward this outcome (Figure 2B). Though ndr1-1 plants were resistant to GA inhibitor paclobutrazol (PAC) at physiologically relevant concentrations, the mutant plants responded to external GA application, which further accelerated its flowering time indicative of a functionally responsive GA signaling pathway. When the ndr1-1 mutant and wild type plants were treated simultaneously with GA and PAC, a reversion to wild type level in flowering time was observed for the ndr1-1 mutants indicating that the GA biosynthesis, not perception was affected. At the same time, Verticillium infection further accelerated the bolting in ndr1-1 plants (Figure 2A middle and bottom panel), suggesting that the pathogen infection promoted a faster transition to the reproductive stage while compromising resistance to the pathogen caused by the genetic lesion in this mutant. Furthermore, work using this GA signaling enhanced ndr1-1 mutant appears to support a model, where inhibition of GA signaling might lead to enhanced resistance to Verticillium.

Mutation in NDR1 renders Arabidopsis defective in the accumulation of SA and potentiation of SAR in the face of a pathogen challenge (Century et al., 1995). Thus, the reduced defense response accompanied by an early transition to flowering in a GA-dependent manner in the ndr1-1 mutant seems to further add to the assumption that resistance to pathogen comes at the cost of normal plant growth and development (Figures 1, 2). This work points toward an important role of GA signaling during Verticillium wilt disease development.

\section{Role of Auxin in Verticillium Resistance}

The expression levels of the auxin receptor genes transport inhibitor response 1 (TIR1), auxin signaling $F$ box protein 1 (AFB1), and auxin signaling $F$ box protein 3 (AFB3) as well as that of the auxin transporter gene auxin resistant 4 (AXR4) were upregulated in $A$. thaliana roots following infection by $V$. dahliae. However, the levels of indole-3-acetic acid (IAA) between the control and the pathogen-treated wild type A. thaliana 
A

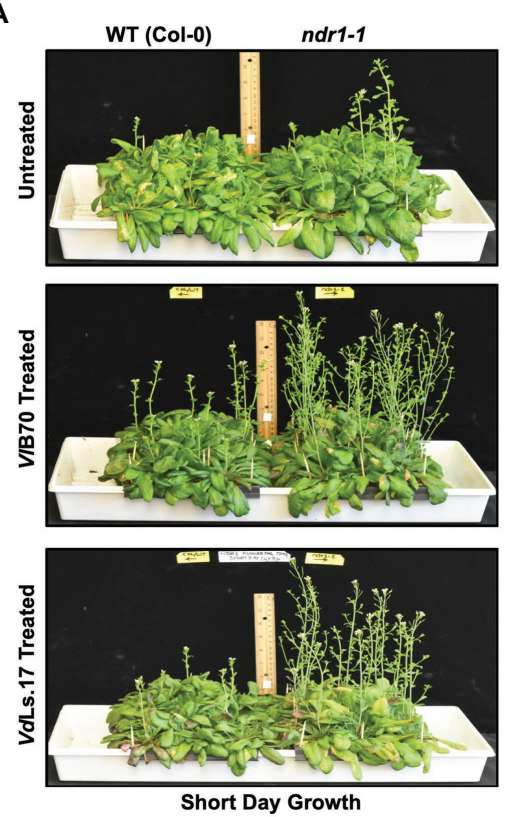

B

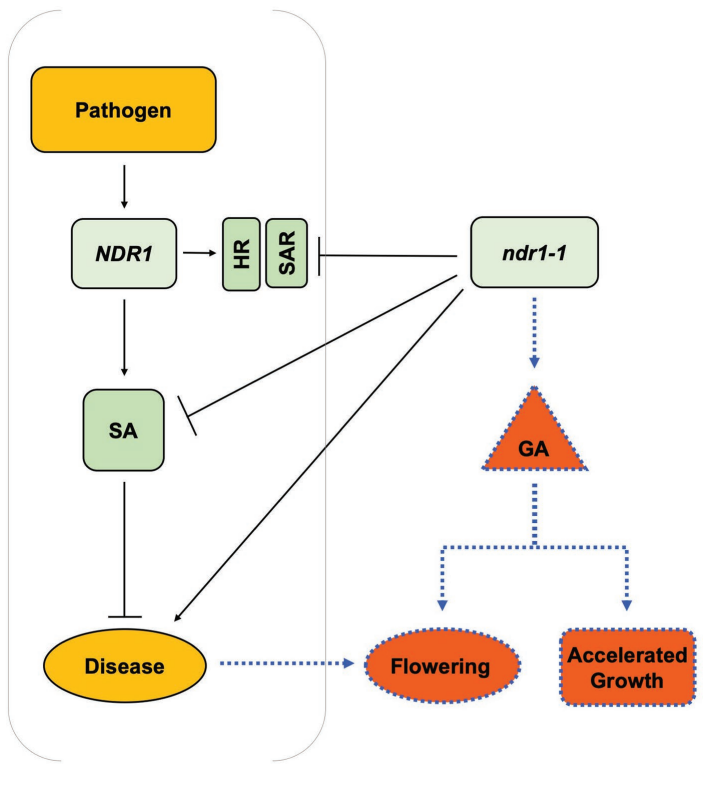

FIGURE 2 | Verticillium-mediated acceleration in growth and flowering time in Arabidopsis, and the role of NDR1 in mediating hormone cross-talk affecting the growth and defense response. (A) Arabidopsis ndr1-1 mutation causes early flowering in comparison to the wild-type Col-0. ndr1-1 plants grown under short day conditions (10/14 h light/dark cycle) flower early and bolt faster relative to the wild-type Col-0 control plants. A similar phenotype occurs in plants grown under the long day conditions (14/10 h light/dark cycle). Reproduced with permission (Dhar et al., 2019). Arabidopsis ndr1-1 mutants are susceptible to Verticillium longisporum (VIBob70) and flower earlier than the corresponding uninfected plants. Plants of both genotypes were grown under short day conditions and were treated with VIBob70 at a concentration of $5 \times 10^{6}$ conidia/ml applied directly to the soil in the 2nd week of their growth. Picture was taken at 4 weeks post infection. Arabidopsis ndr1-1 mutants are susceptible to Verticillium dahliae (VdLs17) and display flowering earlier than the corresponding uninfected plants. Plants were grown, inoculated, and the picture was taken as described for VIBob70. (B) The ndr1-1 mutant as a tool to dissect hormone cross-talk during pathogen infection. The nonhost specific disease resistance 1 (NDR1) gene is required for defense against biotic and abiotic stresses various plant species. It has also been shown to play an important role in defense and altered growth and development response to infection by pathogenic Verticillium sp. While the Arabidopsis ndr1-1 plants are defective in SA signaling rendering the plant susceptible to pathogenic Verticillium, on the one hand, it leads to early senescence and accelerated growth in a GA-dependent manner, on the other hand (Dhar et al., 2019), thus providing an excellent example of the "growth versus defense" response during pathogen attack. SA, salicylic acid; HR, hypersensitive response; SAR, systemic acquired resistance; GA, gibberellic acid; ndr1-1, nonhost specific disease resistance 1-1 mutant.

plants were not significantly different. Interestingly, decreased symptom severity and increased resistance to the pathogen occurred in the pathogen treated $a f b 1, a f b 3$, and axr4 mutant plants. The increased resistance to the pathogen in $a f b 1$ and axr4 was attributed to the upregulation of the defense-related PR1 (a component of the SA pathway). In contrast, the pathogen resistance in the afb3 mutant was attributed to an increased level of PDF1.2 expression (a component of the JA pathway; Fousia et al., 2018). Thus, manipulation of auxin signaling and not its biosynthesis in the root system of $V$. dahliae infected plants may promote Verticillium wilt in susceptible hosts. The activation of the auxin signaling pathway in plant roots might potentially be used as a strategy by the pathogen to suppress the SA dependent host defense responses during the initial biotrophic phase of $V$. dahliae (a hemibiotroph).

In a separate study, 19 strains from 10 Verticillium spp. showed that volatile compounds (VC) from Verticillium caused preferential allocation of resources to drive root growth over shoot growth by manipulating auxin (AUX) signaling pathways. Inhibition of the AUX signaling pathway using an auxin efflux inhibitor compromised this change in growth pattern, underscoring the role of AUX during Verticillium wilt. Furthermore, several components of the AUX signaling pathway, including TIR1, TIR3, AUX1, and AXR1 were involved in the regulation of VC-mediated plant growth (Li et al., 2018a).

\section{Role of Nitric Oxide and Other Growth-Promoting Substances in Verticillium Resistance}

A preinoculative soil drenching with $\beta$-aminobutyric acid (BABA) induced resistance to $V$. longisporum infection of a susceptible oilseed rape (B. napus) cultivar and prevented plant stunting as the disease progressed (Kamble et al., 2013). An early but significant increase in the activity of PAL led to higher synthesis and accumulation of phenylpropanoids, as evidenced by an higher number of cells surrounding the xylem vessels storing phenol was proposed to underlie this chemically induced resistance. This change in vascular architecture, in turn, led to the containment of the pathogen by inhibiting colonization of the shoot (Kamble et al., 2013).

$\mathrm{NO}$ is a gaseous hormone that is mostly implicated in ROS and defense signaling in plants. Verticillium infection and $\mathrm{Vd}$ 
toxins induce cell death with the active involvement of ROS (Jia et al., 2007; Zhang et al., 2013), while Vd toxin alters hormone balances during Verticillium infection (Pegg and Brady, 2002). Both $\mathrm{NO}$ and $\mathrm{H}_{2} \mathrm{O}_{2}$ are produced in cotton suspension cells treated with $V d$ toxin (Jia et al., 2007). Nitric oxide synthase (NOS) and nitrate reductase (NR) enzymes have been implicated in the production of $\mathrm{NO}$ in plants. $\mathrm{Vd}$ toxin induces NO in the leaves of Arabidopsis plants with peak activity around an hour post-treatment though this induction was strongly suppressed in the NR deficient nia1 $x$ nia2 mutant (Shi and $\mathrm{Li}, 2008)$. Further, the $V d$ toxin exerted its cell death-inducing effect through alterations of cytoskeleton and nucleoli. NO production induced by $V d$ toxin caused depolymerization and destabilization of the cortical microtubules rather than the actin microfilaments inside the cell, accompanying an induced cell death resembling HR that coincides with the activation of defense response in the host cells (Shi et al., 2009). While this is an interesting example, few Verticillium effectors are known to actively interfere in changing the hormone levels in the host plants (Liu et al., 2014; Qin et al., 2018).

Results from studies involving VIGS-mediated silencing of a coiled-coil (CC)-NBS-LRR-type gene ( $G b R V d$ ) from a resistant cotton cultivar rendered the cotton plants susceptible to $V$. dahliae infection by downregulation of $\mathrm{SA}, \mathrm{NO}$, and $\mathrm{H}_{2} \mathrm{O}_{2}$ production (Yang et al., 2016). Another study utilizing $V d$ toxins and mutants blocked nitrate reductase-mediated NO production, demonstrating that $\mathrm{H}_{2} \mathrm{O}_{2}$ functions upstream of $\mathrm{NO}$ to modulate the dynamic microtubule cytoskeleton and leading to defense activation during $V$. dahliae infection through the activity of nitrate reductase (Yao et al., 2012).

\section{SUMMARY}

Verticillium wilts result in alterations in the hormone signaling pathways that in turn impact plant development, reproduction, and metabolism, as well as responses to pathogen infection.

Host defense responses to Verticillium infection involve components of hormone signaling pathways, which may act in synergistic or antagonistic ways and result in different outcomes. Furthermore, the nature of this interaction between hormone pathways depends on the organ and stage of the infection process. Nonetheless, there is an overall consensus that SA plays a major role in regulating defense responses and establishing resistance during the initial biotrophic phase of Verticillium spp. infection, while JA signaling is more prominent following the establishment of the pathogen in the vasculature and a switch to necrotrophic lifestyle. Thus, pathway components of either of these hormones seem to affect resistance against Verticillium spp. in an antagonistic manner (Figure 3). In contrast, the role of ET is somewhat intriguing during the disease cycle of Verticillium. While ET perception and signaling is required for normal defense response during the initial stages of the pathogen attack, it seems that Verticillium spp. take advantage of the role of ET in senescence and cell death-promoting processes after switching to a necrotrophic phase to utilize the dying host tissues as a source of nutrients to produce resting structures. The fact that ET signaling plays a major role in promotion and regulation of senescence and during Verticillium infection suggests that an elevated ET signaling may offer an advantage to the host to defend against the pathogen during early stages of infection while this advantage shifts to the pathogen when the host is compromised for the production of resting structures later. Alternatively, the pathogen may trigger ET signaling, accelerating senescence thereby completing its life cycle on senescing and decaying plant tissues (Figure 3). In this respect, establishing an active and direct line of evidence addressing the "cause or effect" conundrum by correlating the lifestyle switch in the pathogen to host developmental senescence, and the exact nature and role of ET signaling in both the pathogen and host will help advance our understanding on this aspect of Verticillium wilt progression.

Though the function of Verticillium effectors and their effect on plant defense have been studied, not much is known about their ability to directly alter/influence hormone signaling in the host plants. However, as summarized in Figure 3, a few studies on the pathogen's ability to directly interfere with host hormone levels are available. Among the known Verticillium effectors that directly influence the host hormone signaling are some that alter the biosynthesis of SA in the host, a few that influence the host BR signaling, and a single one that influences ET signaling (Liu et al., 2014; Qin et al., 2018; Tsolakidou et al., 2020).

Volatiles emitted by pathogenic Verticillium spp. promote growth in Arabidopsis through the AUX signaling pathways (Li et al., 2018a). Additionally, other growth-related hormones such as GA and BR are upregulated during the initial phases of the pathogen attack, suggestive of a response involves a hastening of the propagation of its genetic material by accelerating development when under threat from the pathogen. While many receptor-like kinases (RLK) involved in BR signaling were initially associated with cell growth and development, their role in defense against multiple pathogens has led to an interesting paradigm in terms of "growth versus defense" (Huot et al., 2014). Alternately, this initial spurt in host growth may be stimulated by the pathogen to and the hastening of host growth and remobilizing nutrients under a perceived threat could be subverted to support pathogen growth, colonization, and completion of disease cycle on the host. The identification of the genetic components underlining this "growth versus defense" framework will provide an interesting insight into the pathogenic Verticillium infection strategies since disease symptoms also seem to manifest and overlap with the onset of flowering - a developmental phase marking the transition of the plant from the vegetative to the reproductive state. While the transition to flowering is a progressive developmental phase, it also coincides with the appearance of visible developmental senescence, which in turn also seems to coincide with the manifestation of disease symptoms (Sadras et al., 2000). Thus, resistance to pathogenic Verticillium spp. (involving SA, JA, and ET pathways) seems to be intricately tied with host developmental transition to reproductive phase (mostly involving AUX and GA) and progression of developmental senescence (mostly involving ET and ABA). However, these three fundamental plant processes are coordinated by an intricate network of hormone signaling. Therefore, studies involving hormone relations at an organ-specific and whole plant scale 


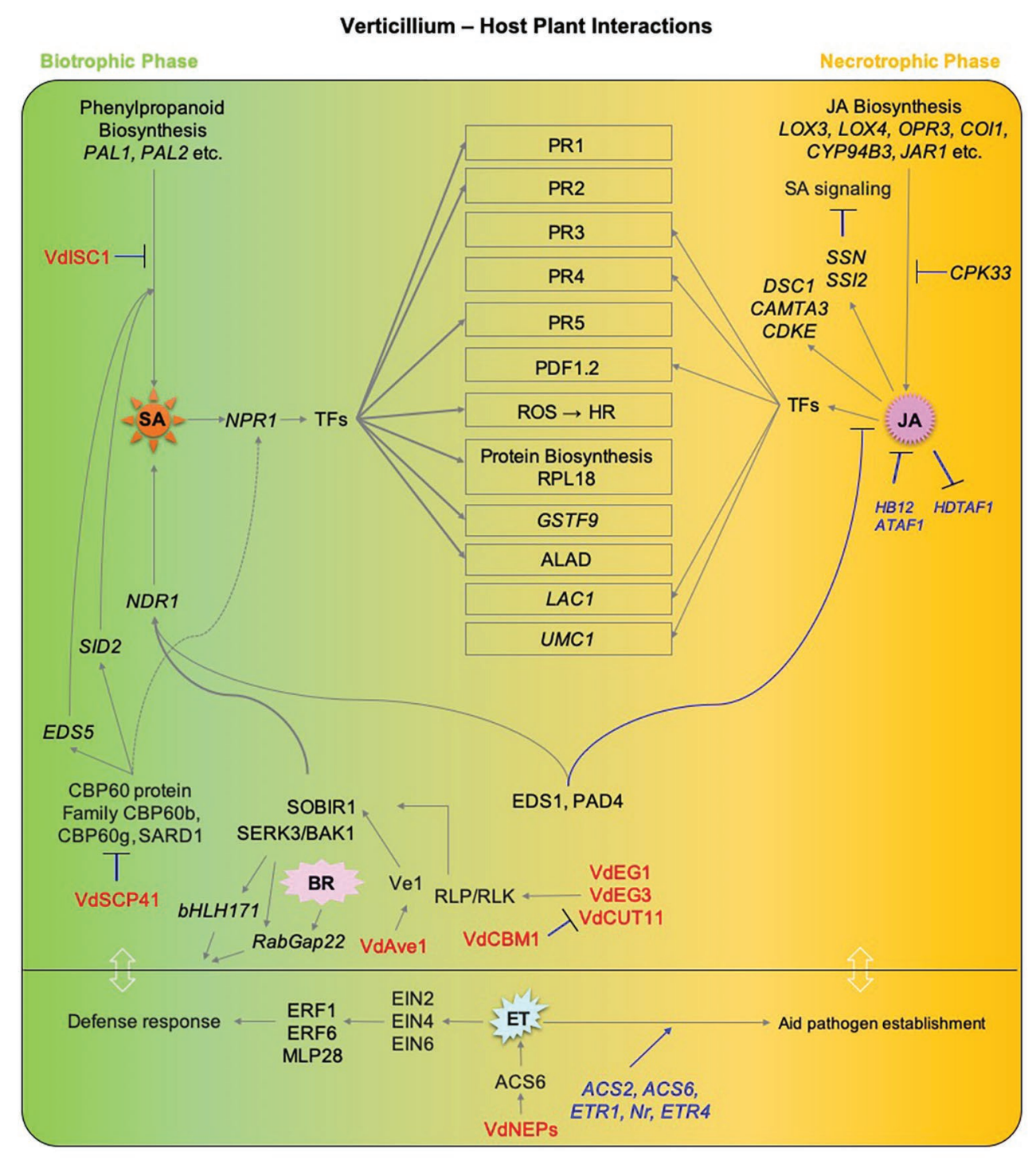

FIGURE 3 | An overview of the major hormone signaling pathways involved in defense responses to Verticillium spp. Components of major hormone signaling pathways at play during Verticillium spp. infection of host plants. Major phytohormone pathways including SA, JA, ET, and BR are affected during the progression of Verticillium wilt. Verticillium dahliae is a hemibiotrophic pathogen that has an initial biotrophic phase (shown in green), following host colonization and death, the pathogen exhibits a necrotrophic lifestyle (shown in orange). SA responses play a major role during the biotrophic phase while JAVET-mediated defenses are largely active later during the switch to necrotrophic phase. Gray lines with arrowheads represent positive regulation, while blue lines with a black head represent negative regulation. Host genetic components involved in negative regulations are shown in blue. Verticillium spp. factors that influence phytohormone pathways directly in the host are shown in red. Components involving studies on PROTEIN are in plain CAPS while those involving GENE are ITALICIZED. TFs, Transcription factors; SA, SALICYLIC ACID; PAL1, PHENYLALANINE AMMONIA-LYASE 1; PAL2, PHENYLALANINE AMMONIA-LYASE 2; NPR1, NONEXPRESSER OF PR GENES 1; SID2, SALICYLIC ACID INDUCTION DEFICIENT 2; EDS1, ENHANCED DISEASE SUSCEPTIBILITY 1; EDS5, ENHANCED DISEASE SUSCEPTIBILITY 5; PAD4, PHYTOALEXIN DEFICIENT 4; NDR1, NON RACE-SPECIFIC DISEASE RESISTANCE 1; ALAD, 5-AMINOLEVULINIC ACID DEHYDRATASE; GSTF9, GLUTATHIONE S-TRANSFERASE PHII 9; SARD1, SAR DEFICIENT 1; CBPGOb, CAM-BINDING PROTEIN 60b; JA, JASMONIC ACID; LOX3, LIPOXYGENASE 3; LOX4, LIPOXYGENASE 4; OPR3, 12-OXOPHYTODIENOATE REDUCTASE 3; OPR4, 12-OXOPHYTODIENOATE REDUCTASE 4; COI1, CORONATINE INSENSITIVE 1; CYP94B3, CYTOCHROME P450_FAMILY 94_SUBFAMILY B_POLYPEPTIDE 3; JAR1, JASMONATE RESISTANT 1; LAC1, LACCASE 1; UMC1, UMECYANIIN-LIKE 1; CPK33, CALCIUM-DEPENDENT PROTEIN KINASE 33; CAMTA3, CALMODULIN-BINDING TRANSCRIPTION ACTINATOR 3; DSC1, DOMINANT SUPPRESSOR OF CAMTA3 1; CDKE, CYCLIN-DEPENDENT KINASE E; SSN, SILENCE-INDUCED STEM NECROSIS; SSI2, SUPPRESSOR OF SA INSENSITIVE 2; HDTF1, HOMEODOMAIN TRANSCRIPTION FACTOR 1; HB12, HOMEOBOX 12; ATAF1, ARABIDOPSIS THALIANA ACTINATING FACTOR 1; ET, ETHYLENE; ETR1, ETHYLENE RESISTANT 1; ETR4, ETHYLENE RESISTANT 4; Nr, NEVER RIPE; ACS2, 1-AMINOCYCLOPROPANE-1CARBOXYLIC ACID SYNTHASE 2; ACS6, 1-AMINOCYCLOPROPANE-1CARBOXYLIC ACID SYNTHASE 6; EIN2, ETHYLENE INSENSITIVE 2; EIN4, ETHYLENE INSENSITIVE 4; EIN6, ETHYLENE INSENSITINE 6; ERF1, ETHYLENE RESPONSE FACTOR 1; ERF6, ETHYLENE RESPONSE FACTOR 6; MPL28, MAJOR LATEX PROTEIN 28; BA, BRASSINOSTEROID; BAK1, BRI1-ASSOCIATED RECEPTOR KINASE; SOBIR1, SUPPRESSOR OF BIR1 1; bHLH171, BHLH-TYPE TRANSCRIPTION FACTOR 171; RABGAP22,

Rab GTPase-ACTIVATING PROTEIN 22; Ve1, VERTICILLIUM Race1 RESISTANCE; Vd, Verticillium dahliae; VdEG1, V. dahliae ENDOGLUCANASE 1; VdEG3, V. dahliae ENDOGLUCANASE 3; VdCUT11, V. dahliae CUTINASE 11; VdCBM1, V. dahlia CARBOHYDRATE-BINDING MODULE FAMILY 1; VdAve1, V. dahliae Avirulence on Ve1; VdSCP41, V. dahliae SECRETORY PROTEIN 41; VdISC1, V. dahliae ISOCHORISMATASE 1; VdNEP, V. dahliae NECROSIS-AND ETHYLENE-INDUCING PROTEIN. 
during the disease cycle will help identify the genetic components and signaling networks that connect growth, defense, senescence, nutrient remobilization, and development during the progression of the Verticillium wilt. The ndr1-1 mutation, which decreases resistance to Verticillium in an otherwise normal plant, leads to early senescence and accelerated flowering (Figure 2A). While lower disease resistance in $n d r 1-1$ has been attributed to a defect in SA signaling (Century et al., 1995), the accelerated flowering is the result of increased GA signaling, an essential component of floral transition or reproductive competency in Arabidopsis (Dhar et al., 2019). This inverse relationship in SA-mediated defense and growth/development promotion by GA strengthens the inverse relationship underlining the "growth versus defense" paradigm. As the ndr1-1 mutation causes susceptibility to pathogens, rapid growth, and early transition to flowering, the plant is perhaps "primed" for pathogen attack as a result of the availability of additional metabolic resources diverted from defense (Figures 2, 4). This line of reasoning seems more likely when considering studies on mutations in genes like $A L A D$ and GhSSN that resulted in increased resistance to Verticillium wilt. Such mutations caused hyperaccumulation of defense-related hormones SA and JA, respectively, while leading to reduced overall growth and developmental defects in the plants (depicted in Figure 4; Sun et al., 2014; Chai et al., 2017).

With successful establishment of the pathogen in a susceptible host, plant growth accelerates initially only to decline progressively as the infection manifests in such host plants. In resistant hosts, the increased tolerance to this vascular pathogen seems to come at the cost of host growth inhibition, with the diversion of resources to fortify host defense responses (Figures 2, 4). Under normal conditions, the BR pathway is a major growth-related hormone pathway that also is involved in defense responses against pathogens. Unsurprisingly current findings support that the steroid phytohormone signaling components, including $B R I 1, B I K 1$, and SOBIR1, are involved in regulating basal defense against other pathogens while contributing to various aspects of plant growth, light responses, and development (Yu et al., 2018).

Evidence of crosstalk between BR-JA and JA-GA is emerging in various systems, which may highlight the "growth-defense" paradigm in Verticillium-host defense. Future helpful studies may address the SA-JA antagonism underlining phase change of the pathogen during infection since this seems to influence the outcome of Verticillium-host interactions. Furthermore, the AUX-ET and AUX-BR synergistic interactions in the roots require greater clarification in response to and during the invasion of Verticillium spp. Defining such hormone interactions and signaling components in a time- and organ-specific manner during various host-Verticillium spp. interactions could help build a unified strategy to approach genetic manipulation of plant hormone signaling networks for broad-spectrum plant defense. Such a development could also set the stage to fabricate designer small molecules/chemical regulators to fortify plant defense or minimize the loss of commercial commodities in crop plants infected by pathogenic Verticillium spp. in conjunction with other traditional and biological disease management methods.

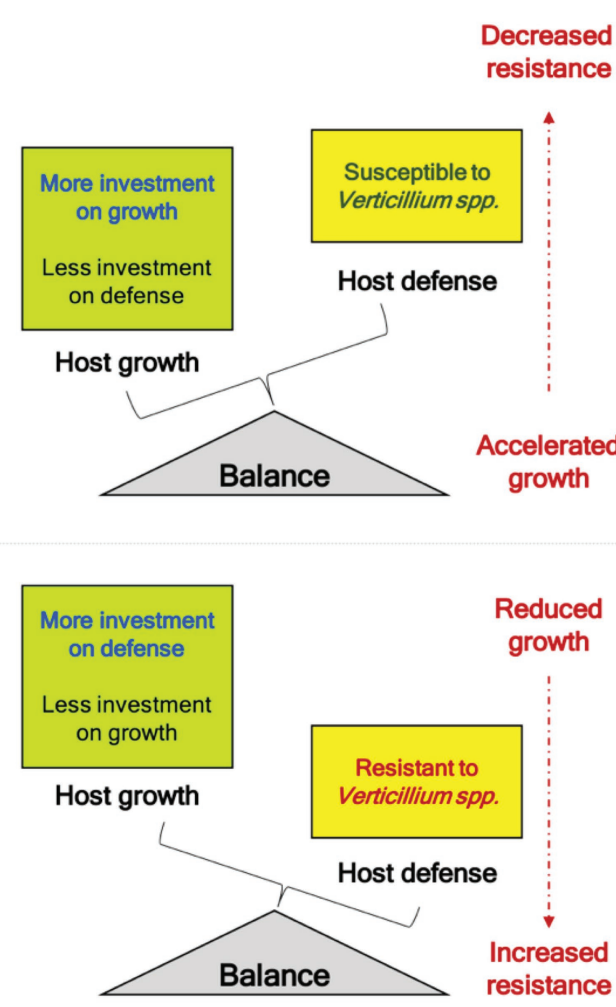

FIGURE 4 | Balance of "growth and defense" during challenge to phytopathogenic Verticillium spp. Studies linking Verticillium resistance to genes like NDR1, ALAD, and GhSSN provide evidence that altered resistance to this soilborne pathogen results in growth defects. Mutations in gene like $A L A D$ and SSN that result in fortified defenses comes at the cost of the plant growth (refer Summary section for details). Alternatively, mutations like ndr1-1 that result in susceptibility to pathogenic Verticillium spp. result in accelerated growth, and faster transition to reproductive competent stage marked by early transition to flowering to propagate the genetic material (refer Figure 2). When the pathogen attack subdues the plant defense responses, an overall irreversible decline in plant growth results with the host succumbing to the pathogen marking the end to a successful infection (susceptibility).

\section{AUTHOR CONTRIBUTIONS}

ND and J-YC prepared all figures. ND wrote the initial draft with significant inputs from SK and KS. SK, KS, and J-YC edited the manuscript. All authors contributed to the article and approved the submitted version.

\section{FUNDING}

ND is funded by a USDA ARS cooperative agreement between SK and KS (University of California, Davis).

\section{ACKNOWLEDGMENTS}

We thank Kelley Clark and Julie Caruna for careful presubmission reviews of the manuscript, as well as for helpful discussions and suggestions. 


\section{REFERENCES}

Aarts, N., Metz, M., Holub, E., Staskawicz, B. J., Daniels, M. J., and Parker, J. E. (1998). Different requirements for EDS1 and NDR1 by disease resistance genes define at least two $R$ gene-mediated signaling pathways in Arabidopsis. Proc. Natl. Acad. Sci. U. S. A. 95, 10306-10311. doi: 10.1073/pnas.95.17.10306

An, C., and Mou, Z. (2013). The function of the mediator complex in plant immunity. Plant Signal. Behav. 8:e23182. doi: 10.4161/psb.23182

Atallah, Z. K., Maruthachalam, K., du Toit, L., Koike, S. T., Davis, R. M., Klosterman, S. J., et al. (2010). Population analyses of the vascular plant pathogen Verticillium dahliae detect recombination and transcontinental gene flow. Fungal Genet. Biol. 47, 416-422. doi: 10.1016/j.fgb.2010.02.003

Berens, M. L., Berry, H. M., Mine, A., Argueso, C. T., and Tsuda, K. (2017). Evolution of hormone signaling networks in plant defense. Annu. Rev. Phytopathol. 55, 401-425. doi: 10.1146/annurev-phyto-080516-035544

Bhat, R. G., and Subbarao, K. V. (1999). Host range specificity in Verticillium dahliae. Phytopathology 89, 1218-1225. doi: 10.1094/PHYTO.1999.89.12.1218

Bibi, N., Ahmed, I. M., Fan, K., Dawood, M., Li, F., Yuan, S., et al. (2017). Role of brassinosteroids in alleviating toxin-induced stress of Verticillium dahliae on cotton callus growth. Environ. Sci. Pollut. Res. Int. 24, 12281-12292. doi: 10.1007/s11356-017-8738-6

Bibi, N., Fan, K., Dawood, M., Nawaz, G., Yuan, S. N., and Wang, X. D. (2014). Exogenous application of epibrassinolide attenuated Verticillium wilt in upland cotton by modulating the carbohydrates metabolism, plasma membrane ATPases and intracellular osmolytes. Plant Growth Regul. 73, 155-164. doi: 10.1007/s10725-013-9877-5

Bowden, R. L., Douglas, I. R., and Sharkey, T. D. (1990). Mechanism of photosynthesis decrease by Verticillium dahliae in potato. Plant Physiol. 94, 1048-1055. doi: 10.1104/pp.94.3.1048

Broekaert, W. F., Delaure, S. L., De Bolle, M. F., and Cammue, B. P. (2006). The role of ethylene in host pathogen interactions. Annu. Rev. Phytopathol. 44, 393-416. doi: 10.1146/annurev.phyto.44.070505.143440

Cao, H., Glazebrook, J., Clarke, J. D., Volko, S., and Dong, X. (1997). The Arabidopsis NPR1 gene that controls systemic acquired resistance encodes a novel protein containing ankyrin repeats. Cell 88, 57-63. doi: 10.1016/ S0092-8674(00)81858-9

Century, K. S., Holub, E. B., and Staskawicz, B. J. (1995). NDR1, a locus of Arabidopsis thaliana that is required for disease resistance to both a bacterial and a fungal pathogen. Proc. Natl. Acad. Sci. U. S. A. 92, 6597-6601. doi: 10.1073/pnas.92.14.6597

Chai, Q., Shang, X., Wu, S., Zhu, G., Cheng, C., Cai, C., et al. (2017). 5-Aminolevulinic acid dehydratase gene dosage affects programmed cell death and immunity. Plant Physiol. 175, 511-528. doi: 10.1104/pp.17.00816

Chini, A., Fonseca, S., Fernandez, G., Adie, B., Chico, J. M., Lorenzo, O., et al. (2007). The JAZ family of repressors is the missing link in JA signaling. Nature 448, 666-671. doi: 10.1038/nature06006

Cronshaw, D. K., and Pegg, G. F. (1976). Ethylene as a toxin synergist in Verticillium wilt of tomato. Physiol. Plant Pathol. 9, 33-34. doi: 10.1016/ 0048-4059(76)90073-4

de Jonge, R., van Esse, H. P., Maruthachalam, K., Bolton, M. D., Santhanam, P., Saber, M. K., et al. (2012). Tomato immune receptor Vel recognizes effector of multiple fungal pathogens uncovered by genome and RNA sequencing. Proc. Natl. Acad. Sci. U. S. A. 109, 5110-5115. doi: 10.1073/pnas.1119623109

Delaney, T. P., Uknes, S., Vernooij, B., Friedrich, L., Weymann, K., Negrotto, D., et al. (1994). A central role of salicylic acid in plant disease resistance. Science 266, 1247-1250. doi: 10.1126/science.266.5188.1247

Deng-wei, J., Min, C., and Qing, Y. (2014). Cloning and characterization of a Solanum torvum NPR1 gene involved in regulating plant resistance to Verticillium dahliae. Acta Physiol. Plant. 36, 2999-3011. doi: 10.1007/s11738-014-1671-0

Derksen, H., Badawi, M., Henriquez, M. A., Zhen, Y., El-Bebany, A. F., and Daayf, F. (2013). Differential expression of potato defense genes associated with the salicylic acid defense signalling pathway in response to weakly and highly aggressive isolates of Verticillium dahliae. J. Phytopathol. 161, 142-153. doi: 10.1111/jph.12038

Dhar, N., Short, D. P. G., Mamo, B. E., Corrion, A. J., Wai, C. M., Anchieta, A., et al. (2019). Arabidopsis defense mutant ndr1-1 displays accelerated development and early flowering mediated by the hormone gibberellic acid. Plant Sci. 285, 200-213. doi: 10.1016/j.plantsci.2019.04.006
Diwan, N., Fluhr, R., Eshed, Y., Zamir, D., and Tanksley, S. D. (1999). Mapping of $V e$ in tomato: a gene conferring resistance to the broad-spectrum pathogen, Verticillium dahliae race 1. Theor. Appl. Genet. 98, 315-319. doi: 10.1007/ s001220051075

Fousia, S., Tsafouros, A., Roussos, P. A., and Tjamos, S. E. (2018). Increased resistance to Verticillium dahliae in Arabidopsis plants defective in auxin signaling. Plant Pathol. 67, 1749-1757. doi: 10.1111/ppa.12881

Fradin, E. F., Abd-El-Haliem, A., Masini, L., van den Berg, G. C. M., Joosten, M. H. A. J., and Thomma, B. P. H. J. (2011). Interfamily transfer of tomato Ve1 mediates Verticillium resistance in Arabidopsis. Plant Physiol. 156, 2255-2265. doi: 10.1104/pp.111.180067

Fradin, E. F., and Thomma, B. P. H. J. (2006). Physiology and molecular aspects of Verticillium wilt diseases caused by $V$. dahliae and V. albo-atrum. Mol. Plant Pathol. 7, 71-86. doi: 10.1111/j.1364-3703.2006.00323.x

Fradin, E. F., Zhang, Z., Ayala, J. C. J., Castroverde, C. D. M., Nazar, R. N., Robb, J., et al. (2009). Genetic dissection of Verticillium wilt resistance mediated by tomato Ve1. Plant Physiol. 150, 320-332. doi: 10.1111/j.1364-3703.2006.00323.x

Gao, X., Li, F., Li, M., Kianinejad, A. S., Dever, J. K., Wheeler, T. A., et al. (2013a). Cotton GhBAK1 mediates Verticillium wilt resistance and cell death. J. Integr. Plant Biol. 55, 586-596. doi: 10.1111/jipb.12064

Gao, W., Long, L., Xu, L., Lindsey, K., Zhang, X., and Zhu, L. (2016). Suppression of the homeobox gene HDTF1 enhances resistance to Verticillium dahliae and Botrytis cinerea in cotton. J. Integr. Plant Biol. 58, 503-513. doi: 10.1111/ jipb. 12432

Gao, W., Long, L., Zhu, L. F., Xu, L., Gao, W. H., Sun, L. Q., et al. (2013b). Proteomic and virus-induced gene silencing (VIGS) analyses reveal that gossypol, brassinosteroids, and jasmonic acid contribute to the resistance of cotton to Verticillium dahliae. Mol. Cell. Proteomics 12, 3690-3703. doi: 10.1074/mcp.M113.031013

Gao, M., Wang, X., Wang, D., Xu, F., Ding, X., Zhang, Z., et al. (2009). Regulation of cell death and innate immunity by two receptor-like kinases in Arabidopsis. Cell Host Microbe 6, 34-44. doi: 10.1016/j.chom.2009.05.019

Gao, X., Wheeler, T., Li, Z., Kenerley, C. M., He, P., and Shan, L. (2011). Silencing GhNDR1 and GhMKK2 compromises cotton resistance to Verticillium wilt. Plant J. 66, 293-305. doi: 10.1111/j.1365-313X.2011.04491.x

Glazebrook, J. (2005). Contrasting mechanisms of defense against biotrophic and necrotrophic pathogens. Annu. Rev. Phytopathol. 43, 220-227. doi: 10.1146/annurev.phyto.43.040204.135923

Goicoechea, N., Aguirreolea, J., Cenoz, S., and Garcia-Mina, J. M. (2000). Verticillium dahliae modifies the concentration of proline, soluble sugars, starch, soluble protein and abscisic acid in pepper plants. Eur. J. Plant Pathol. 106, 19-25. doi: 10.1023/A:1008724816041

Gong, Q., Yang, Z., Wang, X., Butt, H. I., Chen, E., He, S., et al. (2017). Salicylic acid-related cotton (Gossypium arboreum) ribosomal protein GaRPL18 contributes to resistance to Verticillium dahliae. BMC Plant Biol. 17:59. doi: 10.1186/s12870-017-1007-5

Grogan, R. G., Ioannou, N., Schneider, R. W., Small, M. A., and Kimble, K. A. (1979). Verticillium wilt on resistant tomato cultivars in California: virulence of isolates from plants and soil and relationship on inoculum density to disease incidence. Phytopathology 69, 1176-1180. doi: 10.1094/Phyto-69-1176

Gui, Y. J., Chen, J. Y., Zhang, D. D., Li, N. Y., Li, T. G., Zhang, W. -Q., et al. (2017). Verticillium dahliae manipulates plant immunity by glycoside hydrolase 12 proteins in conjunction with carbohydrate-binding module 1. Environ. Microbiol. 19, 1914-1932. doi: 10.1111/1462-2920.13695

Gui, Y. J., Zhang, W. Q., Zhang, D. D., Zhou, L., Short, D. P. G., Wang, J., et al. (2018). A Verticillium dahliae extracellular cutinase modulates plant immune responses. Mol. Plant-Microbe Interact. 31, 260-273. doi: 10.1094/ MPMI-06-17-0136-R

Guo, W., Jin, L., Miao, Y., He, X., Hu, Q., Guo, K., et al. (2016). An ethylene response-related factor, GbERF1-like, from Gossypium barbadense improves resistance to Verticillium dahliae via activating lignin synthesis. Plant Mol. Biol. 91, 305-318. doi: 10.1007/s11103-016-0467-6

Haffner, E., Karlovsky, P., Splivallo, R., Traczewska, A., and Diederichsen, E. (2014). ERECTA, salicylic acid, abscisic acid, and jasmonic acid modulate quantitative disease resistance of Arabidopsis thaliana to Verticillium longisporum. BMC Plant Biol. 14:85. doi: 10.1186/1471-2229-14-85

Hampton, R. E., Wullschleger, S. D., and Oosterhuis, D. M. (1990). Impact of Verticillium wilt on net photosynthesis, respiration and photorespiration in 
field-grown cotton (Gossypium hirsutum). Physiol. Mol. Plant Pathol. 37, 271-280. doi: 10.1016/0885-5765(90)90076-A

Hayes, R. J., McHale, L. K., Vallad, G. E., Truco, M. J., Michelmore, R. W., Klosterman, S. J., et al. (2011). The inheritance of resistance to Verticillium wilt caused by race 1 isolates of Verticillium dahliae in the lettuce cultivar La Brillante. Theor. Appl. Genet. 123, 509-517. doi: 10.1007/s00122-011-1603-y

Hayes, R. J., Vallad, G. E., Qin, Q. M., Grube, R. C., and Subbarao, K. V. (2007). Variation for resistance to Verticillium wilt in lettuce (Lactuca sativa L.). Plant Dis. 91, 439-445. doi: 10.1094/PDIS-91-4-0439

He, X., Wang, T., Zhu, W., Wang, Y., and Zhu, L. (2018b). GhHB12, a HD-ZIP I transcription factor, negatively regulates the cotton resistance to Verticillium dahliae. Int. J. Mol. Sci. 19:3997. doi: 10.3390/ijms19123997

He, X., Zhu, L., Wassan, G. M., Wang, Y., Miao, Y., Shaban, M., et al. (2018a). GhJAZ2 attenuates cotton resistance to biotic stresses via the inhibition of the transcriptional activity of GhbHLH171. Mol. Plant Pathol. 19, 896-908. doi: $10.1111 / \mathrm{mpp} .12575$

He, X., Zhu, L., Xu, L., Guo, W., and Zhang, X. (2016). GhATAF1, a NAC transcription factor, confers abiotic and biotic stress responses by regulating phytohormonal signaling networks. Plant Cell Rep. 35, 2167-2179. doi: 10.1007/s00299-016-2027-6

Hou, X., Lee, L. Y. C., Xia, K., Yan, Y., and Yu, H. (2010). DELLAs modulate jasmonate signaling via competitive binding to JAZs. Dev. Cell 19, 884-894. doi: 10.1016/j.devcel.2010.10.024

Hu, G. S., de Hart, A. K., Li, Y. S., Ustach, C., Handley, V., Navarre, R., et al. (2005). EDS1 in tomato is required for resistance mediated by TIR-class $R$ genes and the receptor-like $R$ gene Ve. Plant J. 42, 376-391. doi: 10.1111/j. 1365-313X.2005.02380.x

Hu, X. -P., Gurung, S., Short, D. P. G., Sandoya, G. V., Shang, W. -J., Hayes, R. J., et al. (2015). Nondefoliating and defoliating strains from cotton correlate with races 1 and 2 of Verticillium dahliae. Plant Dis. 99, 1713-1720. doi: 10.1094/PDIS-03-15-0261-RE

Hu, Q., Min, L., Yang, X., Jin, S., Zhang, L., Li, Y., et al. (2018a). Laccase GhLac1 modulates broad-spectrum biotic stress tolerance via manipulating phenylpropanoid pathway and jasmonic acid synthesis. Plant Physiol. 176, 1808-1823. doi: 10.1104/pp.17.01628

Hu, X., Puri, K. D., Gurung, S., Klosterman, S. J., Wallis, C. M., Britton, M., et al. (2019). Proteome and metabolome analyses reveal differential responses in tomato-Verticillium dahliae-interactions. J. Proteome 207:103449. doi: 10.1016/j.jprot.2019.103449

Hu, Q., Zhu, L., Zhang, X., Guan, Q., Xiao, S., Min, L., et al. (2018b). GhCPK33 negatively regulates defense against Verticillium dahliae by phosphorylating GhOPR3. Plant Physiol. 178, 876-889. doi: 10.1104/pp.18.00737

Huot, B., Yao, J., Montgomery, B. L., and He, S. Y. (2014). Growth-defense tradeoffs in plants: a balancing act to optimize fitness. Mol. Plant 7, 1267-1287. doi: $10.1093 / \mathrm{mp} / \mathrm{ssu} 049$

Inderbitzin, P., Bostock, R. M., Davis, R. M., Usami, T., Platt, H. W., and Subbarao, K. V. (2011). Phylogenetics and taxonomy of the fungal vascular wilt pathogen Verticillium, with the descriptions of five new species. PLoS One 6:e28341. doi: 10.1371/journal.pone.0028341

Inderbitzin, P., and Subbarao, K. V. (2014). Verticillium systematics and evolution: how confusion impedes Verticillium wilt management and how to resolve it. Phytopathology 104, 564-574. doi: 10.1094/PHYTO-11-13-0315-IA

Jia, Z. Q., Yuan, H. Y., and Li, Y. Z. (2007). $\mathrm{NO}$ and $\mathrm{H}_{2} \mathrm{O}_{2}$ induced by Verticillium dahliae toxins and its influence on the expression of GST gene in cotton suspension cells. Chin. Sci. Bull. 52, 1347-1354. doi: 10.1007/s11434-007-0211-3

Johansson, A., Staal, J., and Dixelius, C. (2006). Early responses in the ArabidopsisVerticillium longisporum pathosystem are dependent on NDR1, JA- and ET-associated signals via cytosolic NPR1 and RFO1. Mol. Plant-Microbe Interact. 19, 958-969. doi: 10.1094/MPMI-19-0958

Kamble, A., Koopmann, B., and von Tiedemann, A. (2013). Induced resistance to Verticillium longisporum in Brassica napus by $\beta$-aminobutyric acid. Plant Pathol. 62, 552-561. doi: 10.1111/j.1365-3059.2012.02669.x

Kawchuk, L. M., Hachey, J., Lynch, D. R., Kulcsar, F., van Rooijen, G., Waterer, D. R., et al. (2001). Tomato Ve disease resistance genes encode cell surface-like receptors. Proc. Natl. Acad. Sci. U. S. A. 98, 6511-6515. doi: 10.1073/pnas.091114198

Klosterman, S. J., Atallah, Z. K., Vallad, G. E., and Subbarao, K. V. (2009). Diversity, pathogenicity, and management of Verticillium species. Annu. Rev. Phytopathol. 47, 39-62. doi: 10.1146/annurev-phyto-080508-081748
Klosterman, S. J., Subbarao, K. V., Kang, S., Veronese, P., Gold, S. E., Thomma, B. P. H. J., et al. (2011). Comparative genomics yields insights into niche adaptation of plant vascular wilt pathogens. PLoS Pathog. 7:e1002137. doi: 10.1371/journal. ppat.1002137

Krishna, P. (2003). Brassinosteroid-mediated stress responses. J. Plant Growth Regul. 22, 289-297. doi: 10.1007/s00344-003-0058-z

Kurosawa, E. (1926). Experimental studies on the nature of the substance excreted by the 'bakanae' fungus. Trans. Nat. Hist. Soc. Formosa 16, 213-227.

Li, C., He, X., Luo, X., Xu, L., Liu, L., Min, L., et al. (2014). Cotton WRKY1 mediates the plant defense-to-development transition during infection of cotton by Verticillium dahliae by activating JASMONATE ZIM-DOMAIN1 expression. Plant Physiol. 166, 2179-2194. doi: 10.1104/pp.114.246694

Li, X., Pei, Y., Sun, Y., Liu, N., Wang, P., Liu, D., et al. (2018b). A cotton cyclin-dependent kinase E confers resistance to Verticillium dahliae ediated by Jasmonate-responsive pathway. Front. Plant Sci. 9:642. doi: 10.3389/ fpls.2018.00642

Li, N., Wang, W., Bitas, V., Subbarao, K., Liu, X., and Kang, S. (2018a). Volatile compounds emitted by diverse Verticillium species enhance plant growth by manipulating auxin signaling. Mol. Plant-Microbe Interact. 31, 1021-1031. doi: 10.1094/MPMI-11-17-0263-R

Li, T. -G., Wang, B. -L., Zhang, D. -D., Yin, C. -M., Wang, D., Song, J., et al. (2019). The TIR-NBS-LRR gene GhDSC1 mediates resistance against Verticillium wilt in Gossypium hirsutum. Mol. Plant Pathol. 20, 857-876. doi: $10.1111 / \mathrm{mpp} .12797$

Li, J., Zingen-Sell, I., and Buchenauer, H. (1996). Induction of resistance of cotton plants to Verticillium wilt and of tomato plants to Fusarium wilt by 3 -aminobutyric acid and methyl jasmonate. J. Plant Dis. Prot. 103, 288-299.

Liebrand, T. W. H., van den Berg, G. C. M., Zhang, Z., Smit, P., Cordewener, J. H. G., America, A. H. P., et al. (2013). Receptor-like kinase SOBIR1/EVR interacts with receptor-like proteins in plant immunity against fungal infection. Proc. Natl. Acad. Sci. U. S. A. 110, 10010-10015. doi: 10.1073/pnas. 1220015110

Liu, Y., Huang, X., Li, M., He, P., and Zhang, Y. (2016). Loss-of-function of Arabidopsis receptor-like kinase BIR1 activates cell death and defense responses mediated by BAK1 and SOBIR1. New Phytol. 212, 637-645. doi: 10.1111/ nph.14072

Liu, T., Song, T., Zhang, X., Yuan, H., Su, L., Li, W., et al. (2014). Unconventionally secreted effectors of two filamentous pathogens target plant salicylate biosynthesis. Nat. Commun. 5:4686. doi: 10.1038/ncomms5686

Mansoori, B., and Smith, C. J. (2005). Elicitation of ethylene by Verticillium albo-atrum phytotoxins in potato. J. Phytopathol. 153, 143-149. doi: 10.1111/j. 1439-0434.2005.00943.x

Maruthachalam, K., Atallah, Z. K., Vallad, G. E., Klosterman, S. J., Hayes, R. J., Davis, R. M., et al. (2010). Molecular variation among isolates of Verticillium dahliae and polymerase chain reaction based differentiation of races. Phytopathology 100, 1222-1230. doi: 10.1094/PHYTO-04-10-0122

Misaghi, I., Kosuge, T., and Devay, J. E. (1972). Changes in cytokinin activity associated with development of Verticillium wilt and water stress in cotton plants. Physiol. Plant Pathol. 2, 187-196. doi: 10.1016/0048-4059(72)90001-X

Pantelides, I. S., Tjamos, S. E., and Paplomatas, E. J. (2010a). Insights into the role of ethylene perception in tomato resistance to vascular infection by Verticillium dahliae. Plant Pathol. 59, 130-138. doi: 10.1111/j.1365-3059.2009.02206.x

Pantelides, I. S., Tjamos, S. E., and Paplomatas, E. J. (2010b). Ethylene perception via ETR1 is required in Arabidopsis infection by Verticillium dahliae. Mol. Plant Pathol. 11, 191-202. doi: 10.1111/j.1364-3703.2009.00592.x

Parkhi, V., Kumar, V., Campbell, L. A. M., Bell, A. A., and Rathore, K. S. (2010). Expression of Arabidopsis NPR1 in transgenic cotton confers resistance to non-defoliating isolates of Verticillium dahliae but not the defoliating isolates. J. Phytopathol. 158, 822-825. doi: 10.1111/j.1439-0434.2010.01714.x

Patrick, T. W., Hall, R., and Fletcher, R. A. (1977). Cytokinin levels in healthy and Verticillium-infected tomato plants. Can. J. Bot. 55, 377-382. doi: 10.1139/ b77-048

Pegg, G. F. (1976). The response of ethylene-treated tomato plants to infection by Verticillium albo-atrum. Physiol. Plant Pathol. 9, 215-226. doi: 10.1016/ 0048-4059(76)90055-2

Pegg, G. F., and Brady, B. L. (2002). Verticillium wilts. New York: CABI Publishing.

Pegg, G. F., and Cronshaw, D. K. (1976). Ethylene production in tomato plants infected with Verticillium albo-atrum. Physiol. Plant Pathol. 8, 279-295. doi: 10.1016/0048-4059(76)90022-9 
Poulaki, E. G., Tsolakidou, M. -D., Gkizi, D., Pantelides, I. S., and Tjamos, S. E. (2020). The ethylene biosynthesis genes ACS2 and ACS6 modulate disease severity of Verticillium dahliae. Plan. Theory 9:907. doi: 10.3390/plants9070907

Qin, J., Wang, K., Sun, L., Xing, H., Wang, S., Li, L., et al. (2018). The plantspecific transcription factors CBP60g and SARD1 are targeted by a Verticillium secretory protein VdSCP41 to modulate immunity. elife 7:e34902. doi: 10.7554/ eLife.34902

Ratzinger, A., Riediger, N., von Tiedemann, A., and Karlovsky, P. (2009). Salicylic acid and salicylic acid glucoside in xylem sap of Brassica napus infected with Verticillium longisporum. J. Plant Res. 12, 571-579. doi: 10.1007/ s10265-009-0237-5

Reusche, M., Klaskova, J., Thole, K., Truskina, J., Novak, O., Janz, D., et al. (2013). Stabilization of cytokinin levels enhances Arabidopsis resistance against Verticillium longisporum. Mol. Plant-Microbe Interact. 26, 850-860. doi: 10.1094/MPMI-12-12-0287-R

Robison, M. M., Griffith, M., Pauls, K. P., and Glick, B. R. (2001a). Dual role for ethylene in susceptibility of tomato to Verticillium wilt. J. Phytopathol. 149, 385-388. doi: 10.1111/j.1439-0434.2001.tb03867.x

Robison, M. M., Shah, S., Tamot, B., Pauls, K. P., Moffatt, B. A., and Glick, B. R. (2001b). Reduced symptoms of Verticillium wilt in transgenic tomato expressing a bacterial ACC deaminase. Mol. Plant Pathol. 2, 135-145. doi: 10.1046/j. 1364-3703.2001.00060.x

Roos, J., Bejai, S., Oide, S., and Dixelius, C. (2014). RabGAP22 is required for defense to the vascular pathogen Verticillium longisporum and contributes to stomata immunity. PLoS One 9:e88187. doi: 10.1371/journal. pone. 0088187

Sadras, V. O., Quiroz, F., Echarte, L., Escande, A., and Pereyra, V. R. (2000). Effect of Verticillium dahliae on photosynthesis, leaf expansion and senescence of field-grown sun flower. Ann. Bot. 86, 1007-1015. doi: 10.1006/anbo.2000.1267

Sandoya, G. V., Gurung, S., Short, D. P. G., Subbarao, K. V., Michelmore, R. M., and Hayes, R. J. (2016). Genetics of resistance in lettuce to races 1 and 2 of Verticillium dahliae from different host species. Euphytica 213:20. doi: 10.1007/s10681-016-1813-0

Santhanam, P., van Esse, H. P., Albert, I., Faino, L., Nürnberger, T., and Thomma, B. P. H. J. (2013). Evidence for functional diversification within a fungal NEP1-like protein family. Mol. Plant-Microbe Interact. 26, 278-286. doi: 10.1094/MPMI-09-12-0222-R

Scholz, S. S., Schmidt-Heck, W., Guthke, R., Furch, A. C. U., Reichelt, M., Gershenzon, J., et al. (2018). Verticillium dahliae-Arabidopsis interaction causes changes in gene expression profiles and jasmonate levels on different time scales. Front. Microbiol. 9:217. doi: 10.3389/fmicb.2018.00217

Shi, F. M., and Li, Y. Z. (2008). Verticillium dahliae toxins-induced nitric oxide production in Arabidopsis is major dependent on nitrate reductase. BMB Rep. 41, 79-85. doi: 10.5483/bmbrep.2008.41.1.079

Shi, F. M., Yao, L. L., Pei, B. L., Zhou, Q., Li, X. L., Li, Y., et al. (2009). Cortical microtubule as a sensor and target of nitric oxide signal during the defense responses to Verticillium dahliae toxins in Arabidopsis. Plant Cell Environ. 32, 428-438. doi: 10.1111/j.1365-3040.2009.01939.x

Shigenaga, A. M., and Argueso, C. T. (2016). No hormone to rule them all: interactions of plant hormones during the responses of plants to pathogens. Semin. Cell Dev. Biol. 56, 174-189. doi: 10.1016/j.semcdb.2016.06.005

Short, D. P. G., Sandoya, G., Vallad, G. E., Koike, S. T., Xiao, C. -L., Wu, B. -M., et al. (2015). Dynamics of Verticillium species microsclerotia in field soils in response to fumigation, cropping patterns, and flooding. Phytopathology 105, 638-645. doi: 10.1094/PHYTO-09-14-0259-R

Shu-Ling, Z., Xing-Fen, W., Yan, Z., Jian-Feng, L., Li-Zhu, W., Dong-Mei, Z., et al. (2012). GbWRKY1, a novel cotton (Gossypium barbadense) WRKY gene isolated from a bacteriophage full-length cDNA library, is induced by infection with Verticillium dahliae. Indian J. Biochem. Biophys. 49, 405-413.

Studt, L., Schmidt, F. J., Jahn, L., Sieber, C. M. K., Connolly, L. R., Niehaus, E. -M., et al. (2013). Two histone deacetylases, FfHdal and FfHda2, are important for Fusarium fujikuroi secondary metabolism and virulence. Appl. Environ. Microbiol. 79, 7719-7734. doi: 10.1128/AEM.01557-13

Sun, L., Zhu, L., Xu, L., Yuan, D., Min, L., and Zhang, X. (2014). Cotton cytochrome $P 450$ CYP82D regulates systemic cell death by modulating the octadecanoid pathway. Nat. Commun. 5:5372. doi: 10.1038/ncomms6372

Tjamos, S. E., Flemetakis, E., Paplomatas, E. J., and Katinakis, P. (2005). Induction of resistance to Verticillium dahliae in Arabidopsis thaliana by the biocontrol agent K-165 and pathogenesis-related proteins gene expression. Mol. Plant-Microbe Interact. 18, 555-561. doi: 10.1094/MPMI-18-0555

Tsolakidou, M. D., Pantelides, L. S., Tzima, A. K., Kang, S., Paplomatas, E. J., and Tsaltas, D. (2020). Disruption and overexpression of the gene encoding ACC (1-aminocyclopropane-1-carboxylic acid) deaminase in soil-borne fungal pathogen Verticillium dahliae revealed the role of ACC as a potential regulator of virulence and plant defense. Mol. Plant-Microbe Interact. 32, 639-653. doi: 10.1094/MPMI-07-18-0203-R

Usami, T., Momma, N., Kikuchi, S., Watanabe, H., Hayashi, A., Mizukawa, M., et al. (2017). Race 2 of Verticillium dahliae infecting tomato in Japan can be split into two races with differential pathogenicity on resistant rootstocks. Plant Pathol. 66, 230-238. doi: 10.1111/ppa.12576

Vallad, G. E., Bhat, R. G., Koike, S. T., Ryder, E. J., and Subbarao, K. V. (2005). Weedborne reservoirs and seed transmission of Verticillium dahliae in lettuce. Plant Dis. 89, 317-324. doi: 10.1094/PD-89-0317

Vallad, G. E., Qin, Q. M., Grube, R., Hayes, R. J., and Subbarao, K. V. (2006). Characterization of race-specific interactions among isolates of Verticillium dahliae pathogenic on lettuce. Phytopathology 96, 1380-1387. doi: 10.1094/ PHYTO-96-1380

Vlot, A. C., Dempsey, D. A., and Klessig, D. F. (2009). Salicylic acid, a multifaceted hormone to combat disease. Annu. Rev. Phytopathol. 47, 177-206. doi: 10.1146/annurev.phyto.050908.135202

Wang, Z. -Y., Bai, M. -Y., Oh, E., and Zhu, J. -Y. (2012). Brassinosteroid signaling network and regulation of photomorphogenesis. Annu. Rev. Genet. 46, 701-724. doi: 10.1146/annurev-genet-102209-163450

Wang, J. Y., Cai, Y., Gou, J. Y., Mao, Y. B., Xu, Y. H., Jiang, W. H., et al. (2004). VdNEP, an elicitor from Verticillium dahliae, induces cotton plant wilting. Appl. Environ. Microbiol. 70, 4989-4995. doi: 10.1128/AEM.70. 8.4989-4995.2004

Xie, D. X., Feys, B. F., James, S., Nieto-Rostro, M., and Turner, J. G. (1998). COI1: an Arabidopsis gene required for jasmonate-regulated defense and fertility. Science 280, 1091-1094. doi: 10.1126/science.280.5366.1091

Yan, Z., Xingfen, W., Wei, R., Jun, Y., and Zhiying, M. (2016). Island cotton enhanced disease susceptibility 1 gene encoding a lipase-like protein plays a crucial role in response to Verticillium dahliae by regulating the SA level and $\mathrm{H}_{2} \mathrm{O}_{2}$ accumulation. Front. Plant Sci. 7:1830. doi: 10.3389/fpls.2016.01830

Yang, C. L., Liang, S., Wang, H. Y., Han, L. B., Wang, F. X., Cheng, H. Q., et al. (2015). Cotton major latex protein 28 functions as a positive regulator of the ethylene responsive factor 6 in defense against Verticillium dahliae. Mol. Plant 8, 399-411. doi: 10.1016/j.molp.2014.11.023

Yang, J., Ma, Q., Zhang, Y., Wang, X., Zhang, G., and Ma, Z. (2016). Molecular cloning and functional analysis of GbRVd, a gene in Gossypium barbadense that plays an important role in conferring resistance to Verticillium wilt. Gene 575, 687-694. doi: 10.1016/j.gene.2015.09.046

Yao, L. L., Pei, B. L., Zhou, Q., and Li, Y. Z. (2012). NO serves as a signaling intermediate downstream of $\mathrm{H}_{2} \mathrm{O}_{2}$ to modulate dynamic microtubule cytoskeleton during responses to VD-toxins in Arabidopsis. Plant Signal. Behav. 7, 174-177. doi: 10.4161/psb.18768

Yu, M. -H., Zhao, Z. -Z., and He, J. -X. (2018). Brassinosteroid signaling in plant-microbe interactions. Int. J. Mol. Sci. 19:4091. doi: 10.3390/ijms19124091

Zhang, Y., Wang, X., Li, Y., Wu, L., Zhou, H., Zhang, G., et al. (2013). Ectopic expression of a novel Ser/Thr protein kinase from cotton (Gossypium barbadense), enhances resistance to Verticillium dahliae infection and oxidative stress in Arabidopsis. Plant Cell Rep. 32, 1703-1713. doi: 10.1007/s00299013-1481-7

Zhang, D. D., Wang, J., Wang, D., Kong, Z. Q., Zhou, L., Zhang, G. Y., et al. (2019). Population genomics demystifies the defoliation phenotype in the plant pathogen Verticillium dahliae. New Phytol. 222, 1012-1029. doi: 10.1111/ nph.15672

Zhen, X. H., and Li, Y. Z. (2004). Ultrastructural changes and location of beta-1,3-glucanase in resistant and susceptible cotton callus cells in response to treatment with toxin of Verticillium dahliae and salicylic acid. J. Plant Physiol. 161, 1367-1377. doi: 10.1016/j.jplph.2004.04.007

Zheng, X., Koopmann, B., and von Tiedemann, A. (2019). Role of salicylic acid and components of the phenylpropanoid pathway in basal and cultivarrelated resistance of oilseed rape (Brassica napus) to Verticillium longisporum. Plan. Theory 8:491. doi: 10.3390/plants8110491

Zhou, B. J., Jia, P. S., Gao, F., and Guo, H. S. (2012). Molecular characterization and functional analysis of a necrosis-and ethylene-inducing, protein-encoding 
gene family from V. dahliae. Mol. Plant-Microbe Interact. 25, 964-975. doi: 10.1094/MPMI-12-11-0319

Zhou, Y., Sun, L., Wassan, G. M., He, X., Shaban, M., Zhang, L., et al. (2019). GbSOBIR1 confers Verticillium wilt resistance by phosphorylating the transcriptional factor GbbHLH171 in Gossypium barbadense. Plant Biotechnol. J. 17, 152-163. doi: 10.1111/pbi.12954

Zhu, S., Gao, F., Cao, X., Chen, M., Ye, G., Wei, C., et al. (2005). The rice dwarf virus $\mathrm{P} 2$ protein interacts with ent-kaurene oxidases in vivo, leading to reduced biosynthesis of gibberellins and rice dwarf symptoms. Plant Physiol. 139, 1935-1945. doi: 10.1104/pp.105.072306

Zhu, W., Gao, E., Shaban, M., Wang, Y., Wang, H., Nie, X., et al. (2018). GhUMC1, a blue copper-binding protein, regulates lignin synthesis and cotton immune response. Biochem. Biophys. Res. Commun. 504, 75-81. doi: 10.1016/j.bbrc.2018.08.128
Conflict of Interest: The authors declare that the research was conducted in the absence of any commercial or financial relationships that could be construed as a potential conflict of interest. Mention of trade names or commercial products in this publication is solely for the purpose of providing specific information and does not imply recommendation or endorsement by the United States Department of Agriculture (USDA). USDA is an equal opportunity provider and employer.

Copyright (c) 2020 Dhar, Chen, Subbarao and Klosterman. This is an open-access article distributed under the terms of the Creative Commons Attribution License (CC BY). The use, distribution or reproduction in other forums is permitted, provided the original author(s) and the copyright owner(s) are credited and that the original publication in this journal is cited, in accordance with accepted academic practice. No use, distribution or reproduction is permitted which does not comply with these terms. 